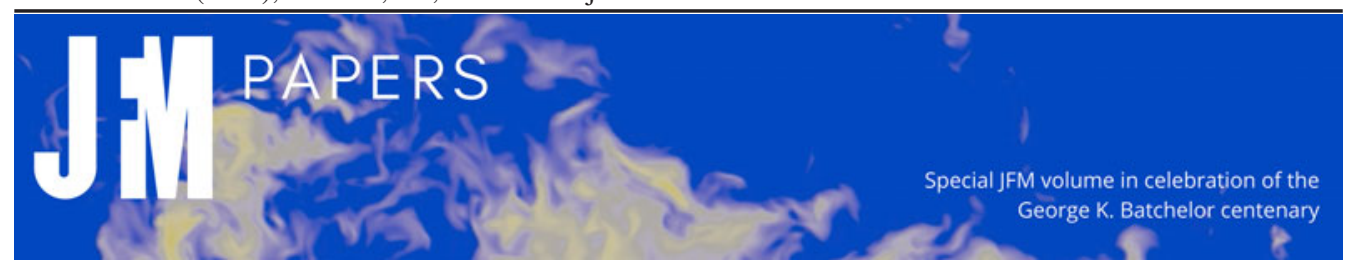

\title{
A metamorphosis of three-dimensional wave structure in transitional and turbulent boundary layers
}

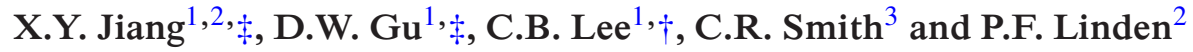 \\ ${ }^{1}$ State Key Laboratory for Turbulence and Complex Systems, College of Engineering, Peking University, \\ No. 5 Yiheyuan Road, Haidian District, Beijing 100871, PR China \\ ${ }^{2}$ Department of Applied Mathematics and Theoretical Physics, Centre for Mathematical Sciences, \\ University of Cambridge, Wilberforce Road, Cambridge CB3 OWA, UK \\ ${ }^{3}$ Department of Mechanical Engineering and Mechanics, Lehigh University, 19 Memorial Drive West, \\ Bethlehem, PA 18015, USA
}

(Received 14 April 2020; revised 16 September 2020; accepted 9 November 2020)

Laminar-turbulent transition in boundary layers is characterized by the generation and metamorphosis of flow structures. However, the process of the evolution from a three-dimensional (3-D) wave to a $\Lambda$-vortex is not fully understood. In order to develop a deeper understanding of the spatiotemporal wave-warping process, we present numerical studies of both K-regime transition and bypass transition. A qualitative comparison of flow visualizations between a K-regime zero pressure gradient (ZPG) case and an adverse pressure gradient (APG) case is done, based on the method of Lagrangian tracking of marked particles. In bypass transition, the development of a 3-D wave packet before the breakdown into a turbulent spot was visualized for both the linear and nonlinear stages. The underlying vortex dynamics was investigated using a proposed method of Lagrangian-averaged enstrophy. The study illustrates that a $\Lambda$-vortex develops from a 3-D warped wave front (WWF), which undergoes multiple folding processes. It is observed that the APG case undergoes a more rapid evolution, precipitating a stronger viscous-inviscid interaction within the boundary layer. It is hypothesized that the amplification and lift-up of a 3-D wave causes the development of high-shear layers and a WWF. In order to seek a relationship between transitional and turbulent boundary layers, Lagrangian methods were also applied to an experimental data set from a turbulent boundary layer at low Reynolds number. Similarity of flow behaviours are observed, which further supports the hypothesis that the amplification of a 3-D wave precipitates low-speed streaks and rotational structures in wall-bounded flows.

Key words: boundary layer structure, transition to turbulence, vortex dynamics

$\dagger$ Email address for correspondence: cblee@ mech.pku.edu.cn

$\$$ These authors contributed equally to this work. 


\section{Introduction}

The origin of wall-bounded turbulence has been one of the most classic research problems for the fluid mechanics community. This problem has attracted much attention because of the fundamental significance in understanding the process of turbulence production and the considerable difficulties encountered in identifying the building blocks of coherent structures in laminar-turbulent transition. There have been many reviews related to the coherent structures in transitional and well-developed turbulent boundary layers (Smith 1984; Robinson 1991; Kachanov 1994; Rempfer 2003; Lee \& Wu 2008; Jiménez 2018; Lee \& Jiang 2019; Marusic \& Monty 2019). However, most research investigating the generation or regeneration of turbulent structures usually does not take into account the early transition stage where three-dimensional (3-D) waves play important roles. The origin of hairpin vortices that many studies take as the dominant flow structure of turbulent boundary layers is not well understood (Marusic 2009). In the present study, a comparative analysis is made between a zero-gradient (Blasius) boundary layer (ZPG) and a Falkner-Skan (FS) flow with an adverse pressure gradient (APG). In addition, a direct numerical simulation (DNS) study of a turbulent spot and an experimentally determined low-Reynolds-number turbulent boundary layer are also presented for comparison with the transitional flows.

In the classical work of Hama and his colleagues, which employed hydrogen bubble visualization to observe structures within a transitional boundary layer (Hama, Long \& Hegarty 1957; Hama \& Nutant 1963), they observed that a Tollmien-Schlichting wave (TS) wave warps three dimensionally during amplification, acquiring a longitudinal vorticity component along its swept-back front. This layer of concentrated high-shear, which appears as an inflection in vertical hydrogen bubble timelines (i.e. a 'kink'), subsequently develops into a hairpin-shaped, discrete vortex. This localized and intensified warped wave front (WWF) was considered as the source of subsequent hairpin-shaped vortices. The warping process and kinked profiles were frequently observed experimentally or numerically in transitional boundary layers (Wortmann 1981; Laurien \& Kleiser 1989; Rist \& Fasel 1995; Lee 1998). Jiang et al. (2020a) systematically investigated the development of a WWF in the early stage of $\mathrm{K}-, \mathrm{N}$ - and O-regime transition, demonstrating qualitative similarity between the stages. However, the roles of a WWF in APG transition are not clear, and the manner in which a WWF originates and evolves in an APG is not well understand. This emphasizes the need to examine the details of the warping process and the development of a WWF prior to the emergence of $\Lambda$-vortices in an APG flow.

A hypothesis based on a 3-D wave-like structure, termed a soliton-like coherent structure (SCS), has been proposed by Lee (1998) to explain the velocity profile 'kink'. Such a 3-D wave-like structure was observed within the whole boundary layer, in both the early and later stages of a transitional boundary layer (Lee \& Li 2007; Lee \& Wu 2008). Chen (2013) confirmed the existence of an SCS and its dominant role in the vortex evolution process using DNS. Zhao, Yang \& Chen (2016) investigated the evolution of material surfaces in channel flow, and determined that a triangular material bulge forms before the roll up of a vortex sheet, which is similar to the results of Chen (2013) and Lee \& Wu (2008). Their simulation shows that the heads of primary hairpin-like structures develop directly from those triangular bulges.

A decelerating FS flow (Hartree parameter $\beta_{H}=-0.18$ ) was investigated by Kloker \& Fasel (1995) using DNS. They observed that the resulting breakdown process for an adverse pressure gradient is dramatically more complex than a K-regime breakdown for a Blasius flow. They observed that a lower characteristic high-shear layer (HSL) 


\section{Three-dimensional wave structure in boundary layers}

formed between the primary $\Lambda$-vortex, and plays a key role in precipitating an ultimate breakdown to turbulence. Borodulin, Kachanov \& Roschektayev (2006) implemented an experimental study of coherent structures for a late-stage APG boundary layer $\left(\beta_{H}=\right.$ $-0.115)$. Formation of $\Lambda$-vortices, $\Lambda$-shaped 3 -D HSLs and ring-like vortices was observed, and were shown to be qualitatively similar to those found in ZPG boundary layers. They pointed out that the instantaneous velocity and vorticity fields seem to correspond qualitatively to those present in the near-wall region of a developed turbulent boundary layer. Lee (2000) discussed the similarity between the physical mechanisms for the structure formation in both transitional and developed turbulent boundary layers, and proposed a universal path to transition based on the concept of SCS. Sayadi, Hamman \& Moin (2013) and Sayadi et al. (2014) evaluated flow statistics and near-wall dynamics for $\mathrm{K}$ - and N-regime breakdown during late stage transition, and proposed late-stage transition, manifested by hairpin packets, as a reduced-order model of a turbulent boundary layer. The key structure that supports the similarity is the low-speed streak (LSS), which plays key roles in turbulence production (Smith et al. 1991; Asai, Minagawa \& Nishioka 2002; Asai et al. 2007). Schoppa \& Hussain (2002) investigated a turbulent channel flow using linear theory and DNS. They identified a streak transient growth (known as STG) mechanism, by which streaks generate streamwise vortices and hence sustained turbulence. However, the collective simulation and experimental studies reached no consensus on the near-wall turbulence production cycle. The relationship between LSS, $\Lambda$ (or hairpin) vortices, and the so called 'bursts' in both transitional and turbulent boundary layers merit further investigation. Turbulent spots can be found frequently in both the bypass transition flow and turbulent flow. Park et al. (2012) investigated turbulent spots and fully developed turbulence by examining the numerical flow data of a flat-plate boundary layer with a passively heated wall, and found little difference in the structure and transport processes between these two cases. Recently, Wu, Moin \& Adrian (2020) observed that two different types of hairpin vortices develop in a pipe flow: reverse hairpin vortices in the near-wall region and forward hairpin vortices in core region. A patch of negative skin friction, which is caused by a reverse hairpin vortex, occurs both in the transitional region and the fully developed turbulent flow region.

The inception mechanism for turbulent spots is a common concern in the study of bypass transition. Wu et al. (2017) observed that the transitional-turbulent spot inception mechanism is analogous to the secondary instability of a transitional boundary layer, with a spot originating from a spanwise vortex filament and a $\Lambda$-vortex. They also observed turbulent-turbulent spots in the inner layer of a developed turbulent boundary layer, which coincide with local concentrations of high levels of enstrophy. Lee \& Wu (2008) pointed out that the turbulent spot is a composite structure, which is a combination of several 3-D wave structures (SCS) and the residual vortices within the spot boundaries. However, there is still a lack of understanding of the process by which a wave packet develops into a turbulent spot.

There are many methods that have been used to study the dynamic process behind the visualized metamorphosis of flow structures, either using Eulerian approaches (Chong, Perry \& Cantwell 1990; Jeong \& Hussain 1995; Tian et al. 2018) or Lagrangian approaches (Haller 2005; Green, Rowley \& Haller 2007). Lagrangian coherent structures (LCS) are distinguished as surfaces of trajectories in a dynamical system that form the skeletons of Lagrangian particle dynamics over a time interval of interest (Haller \& Yuan 2000; Haller 2015). Lagrangian-averaged vorticity deviation (LAVD) is one of the representative LCS detection methods that extract the influence of vortices on nearby flow behaviour (Haller et al. 2016). However, due to the non-uniformity of background vorticity in boundary 


\section{X.Y. Jiang, D.W. Gu, C.B. Lee, C.R. Smith and P.F. Linden}

layers, the value of LAVD may rely on the selected initial fluid volume. Recently, the determination of isosurfaces of the enstrophy field has been applied to stratified flows to identify turbulent/non-turbulent interfaces (known as TNTI) (Neamtu-Halic et al. 2020). The evolution of enstrophy is closely related to vortex stretching effects, which is a critical kinematic mechanism in a shearing process. In the present work, a method of Lagrangian-averaged enstrophy (LAE) is employed to analyse wall-bounded flows, using the integral of the enstrophy (i.e. $|\omega|^{2}$ ) along fluid trajectories over a finite time interval of interest.

The objective of the present paper is to characterize the development of 3-D waves during transition, and to understand the underlying dynamic process that gives rise to $\Lambda$-vortices, especially in APG cases and for turbulent spots. We also examine the connections between transitional flows and turbulent flows. The remainder of this paper is organized as follows: in $\S 2$, the methods of nonlinear parabolized stability equations (NPSE) and DNS are described, including the method of Lagrangian tracking; in $\S 3$, a comparative study of numerical flow visualization and corresponding LCS based on LAE is presented for ZPG and APG cases; in $\S 4$, the development of a 3-D wave packet into a turbulent spot is illustrated, including an analysis of the enstrophy intensity; in $\S 5$, Lagrangian methods, the same as employed for the transition cases, are applied to an experimental data set from a turbulent boundary layer, attempting to understand the streak behaviour in near-wall turbulence; in $\S 6$, the underlying physical mechanisms for the three different flows are discussed; concluding remarks are given in $\S 7$.

\section{Numerical approaches}

\subsection{PSE}

The methods of NPSE is suitable for investigating linear and weakly nonlinear evolution of disturbances. The detailed formulation of NPSE can be found in Bertolotti, Herbert \& Spalart (1992) and Chang \& Malik (1994). The implementation of NPSE in the present work is the same as employed by Jiang et al. (2020a). The code was verified in Chen, Zhu $\&$ Lee (2017), and the details of the operators in the governing equations for the shape functions of each mode can be found in Zhu et al. (2018).

Here, NPSE was utilized to study the flow patterns for K-type transitions, for both a ZPG and an APG. In the language of frequency-wavenumber, the notion $(\omega, \beta)$ is used, where $\omega$ and $\beta$ denote the streamwise frequency and spanwise wavenumber, respectively. For simplicity, $\omega$ and $\beta$ are normalized on the corresponding primary waves. For K-regime transition with a ZPG, a two-dimensional (2-D) TS wave of normalized frequency $F=$ $106 \times 10^{-6}$ (corresponds to period $T=148.2$ ) and a pair of oblique waves at the same frequency were introduced at the inlet $\left(x_{0}=400\right)$ with a relatively smaller amplitude, i.e. modes $(1,0)$ and $(1, \pm 1)$.

For K-regime transition with an APG, a 2-D TS wave of normalized frequency $F=$ $435 \times 10^{-6}$ (corresponding to period $T=56.0$ ) was forced at the inlet by a pair of oblique waves at the same frequency $\left(x_{0}=258\right)$. The profile of an FS boundary layer is characterized by a power-law dependence on the free stream velocity $U_{\infty}$ in the streamwise coordinate direction, i.e. $U_{\infty}(x)=C x^{m}$. The Hartree parameter, related to $m$ by $\beta_{H}=2 m /(1+m) \approx-0.062$, is a measure of the strength of the mean pressure gradient. The detailed information for the wave components forced at the inlet of the simulation for these transition scenarios is shown in table 1. 


\begin{tabular}{|c|c|c|c|c|c|c|c|c|}
\hline \multirow[b]{2}{*}{ Case } & \multicolumn{3}{|c|}{ 2-D wave } & \multicolumn{4}{|c|}{ 3-D wave } & \multirow[b]{2}{*}{ Hartree parameter $\left(\beta_{H}\right)$} \\
\hline & mode & $F \times 10^{6}$ & $\mathcal{A}$ & mode & $F \times 10^{6}$ & $\beta$ & $\mathcal{A}$ & \\
\hline ZPG & $(1,0)$ & 106 & $0.2 \% U_{\infty}$ & $(1, \pm 1)$ & 106 & 0.05 & $0.14 \% U_{\infty}$ & 0 \\
\hline APG & $(1,0)$ & 435 & $4 \% U_{\infty}$ & $(1, \pm 1)$ & 435 & 0.18 & $0.2 \% U_{\infty}$ & -0.062 \\
\hline
\end{tabular}

Table 1. Numerical settings for the two K-regime transition simulations. A normalized notation $(\omega, \beta)$ is used in mode description. The first digit indicates multiples of the fundamental streamwise frequency. The second digit refers to multiples of the fundamental spanwise wavenumber.

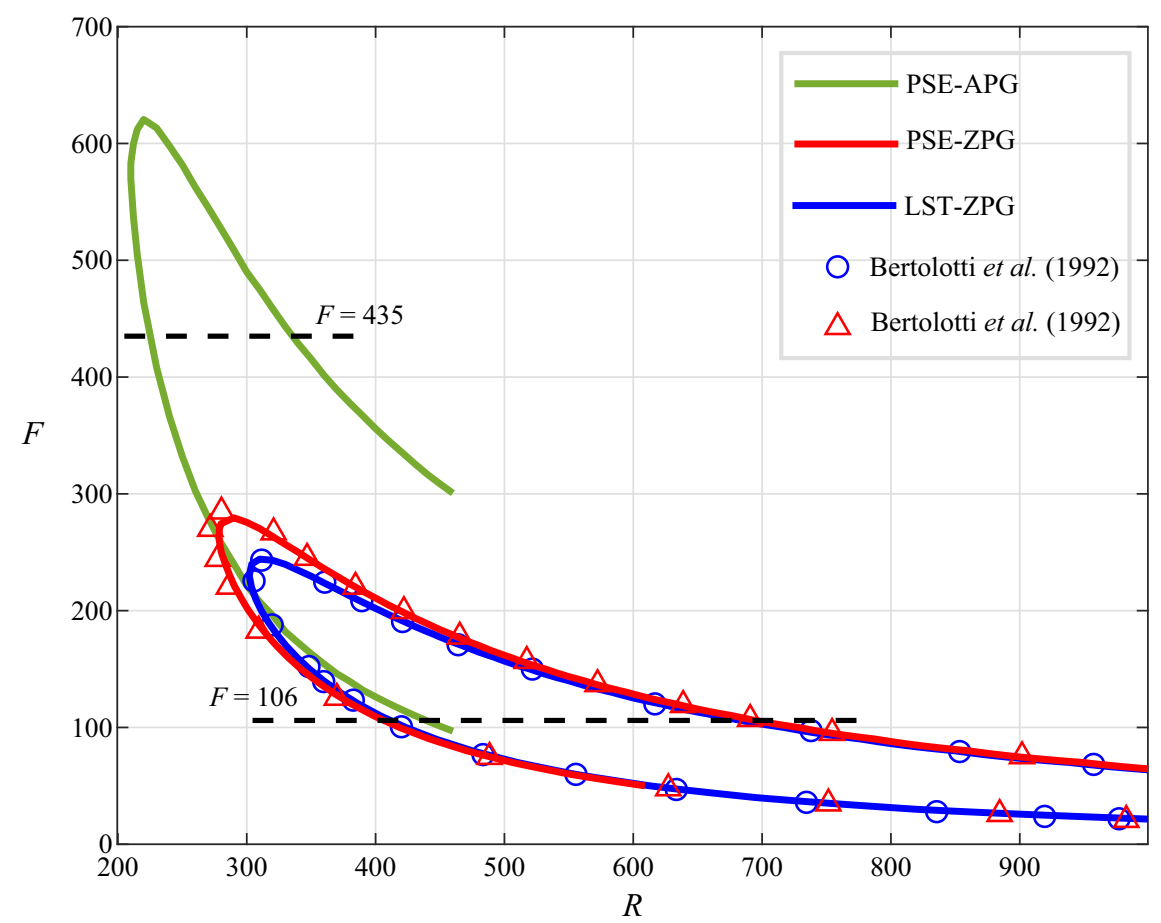

Figure 1. Neutral stability curves for a Blasius boundary layer based on PSE (red line) and linear stability theory (LST) (blue line), and an FS boundary layer based on NPSE (green line). The input normalized frequency for the Blasius boundary layer is $F=106 \times 10^{-6}$. The corresponding data of Bertolotti et al. (1992) are represented by triangles and circles, respectively. The Hartree parameter for the FS boundary layer is $\beta_{H}=-0.062$, with an input normalized frequency of $F=435 \times 10^{-6}$.

The neutral curves for both the ZPG and APG are shown in figure 1. The frequency and amplitude of disturbance input for the two cases are different, thus only a qualitative comparison is made, focusing on the development from 3 -D waves to $\Lambda$-vortices. The corresponding data of Bertolotti et al. (1992) are also represented by triangles and circles, respectively. In figure $1, R=\sqrt{U_{\infty} x^{*} / v}$, where $v$ is the fluid kinematic viscosity, and $x^{*}$ is the physical streamwise distance. Note that $R_{0}=\sqrt{U_{\infty} x_{0}^{*} / v}$ is the value at the inlet position, which is 400 and 258 for ZPG and APG boundary layers, respectively. In parabolized stability equations (PSE), $x=x^{*} / \delta_{0} /\left(2-\beta_{H}\right)$, where $\delta_{0}=\sqrt{\left(\nu x_{0}^{*} / U_{\infty}\right)}$ is the initial thickness of the boundary layer. 


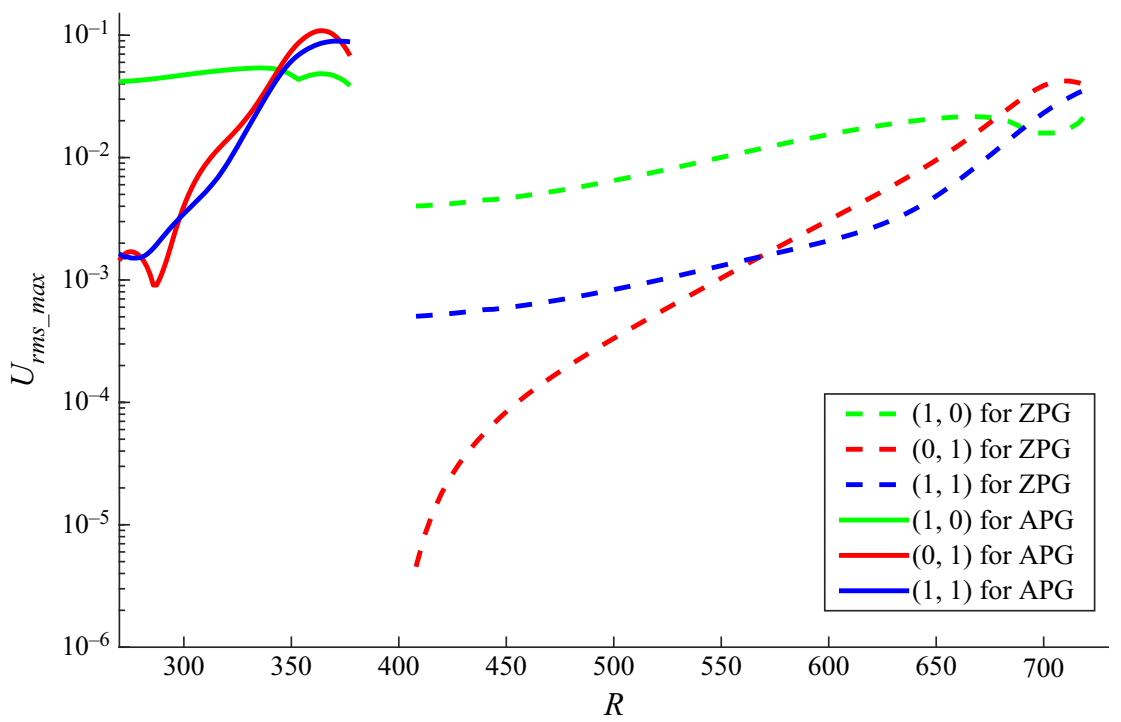

Figure 2. Mode amplitude evolution for K-regime transitions with ZPG (dashed lines) and APG (solid lines).

The development of the $y$-maxima of the selected Fourier amplitudes for both the transitions of ZPG and APG are shown in figure 2. For the ZPG boundary layer, after the initiation of a fundamental 2-D TS wave $(1,0)$ and oblique waves $(1,1)$, streak modes $(0,1)$ progressively develop downstream, exceeding the amplitude of the fundamental disturbance $(1,0)$ at approximately $R=680(x \approx 1156)$. A similar growth in the amplitude of the streak modes and oblique modes is observed for the APG case, the amplitude exceeding the initial characteristics at approximately $R=345(x \approx 224)$. The NPSE simulation for the ZPG case becomes invalid at $R=728(x=1325)$, while the APG simulation becomes invalid at $R=377(x=267)$.

\section{2. $D N S$}

The simulation of bypass transition in this paper is governed by the compressible Navier-Stokes equations, which are listed in appendix A. The code was developed and verified by $\mathrm{Li}, \mathrm{Fu} \& \mathrm{Ma}$ (2008). The calculations were performed on the TianHe 2 at the GuangZhou Supercomputer Center.

A high-order finite-difference method was used in this case. The viscous terms were discretized using an eighth-order central finite-difference scheme. The convection terms were discretized using a seventh-order weighted, essentially non-oscillatory (known as WENO), scheme. For the time step, a third-order accurate Runge-Kutta method was used.

The mesh number (streamwise $\times$ wall normal $\times$ spanwise) is $1600 \times 150 \times 150$, and physical length of the computation zone is $400 \mathrm{~mm} \times 40 \mathrm{~mm} \times 120 \mathrm{~mm}$, respectively. The mesh was refined near the wall surface in the wall normal direction and was kept uniform in the streamwise and spanwise directions. An example schematic of the mesh is shown in figure 3 .

The basic parameters in this case are shown in table 2 , where $R e_{0}$ is the unit Reynolds number, $T_{0}$ is the stagnation temperature and $T_{e}$ is the static temperature. The velocity and temperature are normalized on the inlet free stream; lengths are normalized on $1 \mathrm{~mm}$. 


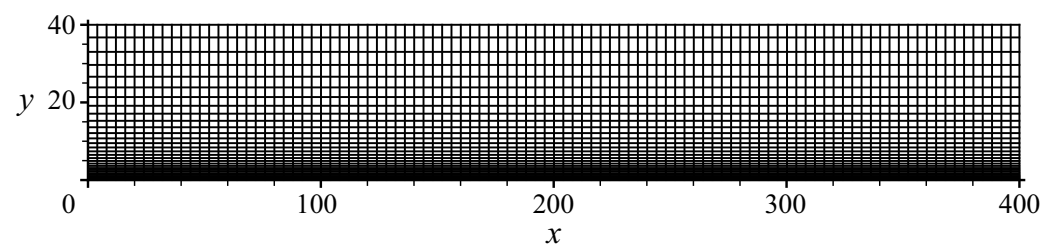

Figure 3. Schematic mesh of DNS.

\begin{tabular}{|c|c|c|c|c|c|c|c|c|c|c|}
\hline$M a$ & $R e_{0} / \mathrm{m}^{-1}$ & $\operatorname{Pr}$ & $T_{0} / \mathrm{K}$ & $T_{e} / \mathrm{K}$ & $x_{b} / \mathrm{mm}$ & $x_{e} / \mathrm{mm}$ & $z_{b} / \mathrm{mm}$ & $z_{e} / \mathrm{mm}$ & $\omega$ & $R_{0} / \mathrm{mm}$ \\
\hline & $6 \times 10^{6}$ & 0.72 & 350 & 125.0 & 8 & 12 & 58 & 62 & 2.09 & 2 \\
\hline
\end{tabular}

Table 2. The DNS parameters.

For boundary conditions, the inflow boundary is set as the laminar flow at $x=10 \mathrm{~mm}$ to reduce the computational cost. Free stream parameters are applied at the upper boundary, and a non-reflecting boundary condition is used for the upper and outflow boundary. No-slip and isothermal conditions are specified at the wall surface, where $T_{\text {wall }} / T_{e}=5$, and the localized disturbance generated by blowing and suction is added at $x \approx 20 \mathrm{~mm}$. A periodic condition is applied in the spanwise direction.

An initial calculation of a laminar flow was specified as the initial flow. To generate a localized wave packet, a disturbance was initiated at $x=10 \mathrm{~mm}$ from the inlet. The function for the disturbance is given by (2.1)

$$
\begin{gathered}
v_{b s}=A_{0} \cos ^{3}\left(\pi x_{0}\right) \cos ^{3}\left(\pi z_{0}\right) \sin \left(-\omega\left(t-t_{s}\right)\right), \quad t_{s}<t<t_{s}+\frac{2 \pi}{\omega} \\
x_{0}=\frac{x-\left(x_{b}+x_{e}\right) / 2}{x_{e}-x_{b}}, \quad z_{0}=\frac{z-\left(z_{b}+z_{e}\right) / 2}{z_{e}-z_{b}} \\
\sqrt{\left(x-\frac{x_{b}+x_{e}}{2}\right)^{2}+\left(z-\frac{z_{b}+z_{e}}{2}\right)^{2}}<r_{0} .
\end{gathered}
$$

Here, $v_{b s}$ is the blowing or suction velocity at the wall surface; $A_{0}$ denotes the amplitude of the disturbance, and is set to 0.05 for this case; parameter $t_{s}$ represents the start time of the simulation, and is 0 for this case. The disturbance is only applied for a time of $2 \pi / \omega$, where $\omega$ is the disturbance frequency. The subscript ' $b$ ' denotes where the disturbance begins and ' $e$ ' denotes where the disturbance ends; $r_{0}$ is the radius of the circle where the disturbance was initiated, and is $2 \mathrm{~mm}$ in this case.

\subsection{Method of Lagrangian tracking of marked particles}

In this paper, results are primarily based on marked particles which are followed using Lagrangian tracking methods. Flow visualizations that are reconstructed from the NPSE velocity field data are termed 'NPSE visualization' (Jiang et al. 2020a). A brief introduction to the Lagrangian tracking method employed is given here.

The trajectory of a marked particle is also called a pathline, which is determined by integrating (2.4) over real time,

$$
V(X(t), t)=\mathrm{d} X(t) / \mathrm{d} t .
$$


(a)

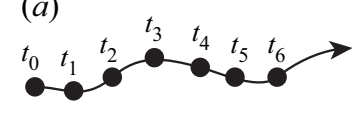

(b)

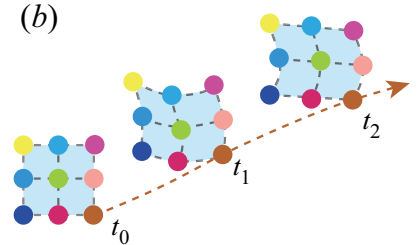

(c)

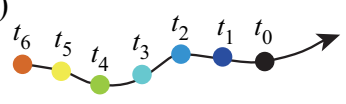

(d)

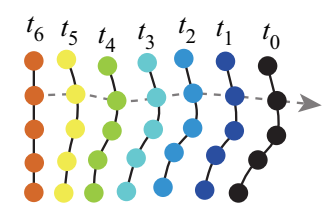

Figure 4. Lagrangian tracking of marked particles: $(a)$ pathline; $(b)$ tracking of a material surface consisting a grid of particles; $(c)$ streakline; $(d)$ timelines.

A pathline provides information on the positional history of a specific particle, as shown in figure 4(a). If a grid of particles is traced, the evolution of a material surface that is comprised of the particles can be obtained, as shown in figure 4(b). A streakline provides a more intuitive physical view than a pathline, because it consists of a series of fluid particles that are repeatedly released from a fixed location. Figure 4(c) shows a streakline at $t=t_{6}$ which includes seven particles in succession. The position of each particle at $t_{6}$ is calculated from (2.4) over a time interval $\left[t_{i}, t_{6}\right]$, where the subscript $i$ indicates the release time of the particle. Timelines are lines connecting a series of particles released from adjacent locations at the same time. A timeline pattern, similar to a hydrogen bubble visualization, is shown in figure $4(d)$, which is generated by a series of periodically released timelines. Streaklines can also be illustrated by connecting sequential particles released from the same location, as illustrated by the dashed line in figure $4(d)$.

\section{K-type transition with ZPG and APG}

\subsection{Numerical flow visualization}

The typical pattern for the K-regime is characterized as peak and valley regions, with structures of 3-D waves and $\Lambda$-vortices aligning in the streamwise direction. In order to illustrate the structure development from a 2-D TS wave to a $\Lambda$-vortex, timelines were initiated at a near-wall position, $y=1.71$, with a spanwise range covering a whole peak region. Figure 5(a) shows the evolution of flow structures for K-regime transition with ZPG from $x=1011.2$. The colour of the particles indicates the wall-normal distance from the wall. Figure 5(a) clearly shows that a $\Lambda$-vortex appears at $x \approx 1250$, well downstream of a 3-D WWF. According to hydrogen bubble studies by Hama \& Nutant (1963), the deformation of the band of accumulated particles represents the deformation of the vorticity field. The concentration of particles occurs initially at the front of the 2-D TS wave, then deforms laterally, creating a 3-D WWF, and finally evolves into the legs of a $\Lambda$-vortex. Note that the tip of the $\Lambda$-vortex is observable in figure 5(a), rather than as an 'open' $\Lambda$-shaped vortex, as observed in some investigations (Hama \& Nutant 1963; Wortmann 1981; Lee \& Wu 2008). There are several regions where the timelines are strongly retarded, labelled with a $\mathrm{K}$ in figure $5(a)$, located near the bottom of the 3-D WWF and the $\Lambda$-vortex. These low-speed regions are considered to illustrate the formation of an incipient transition streak (Jiang et al. 2020a).

For K-regime transition with an APG, figure 5(b) shows a qualitatively similar timeline pattern (initiated at $x=164.5$ ), including the development of a $\Lambda$-vortex, albeit much more intense and rapidly developing than the ZPG case. However, the back-swept HSL of 


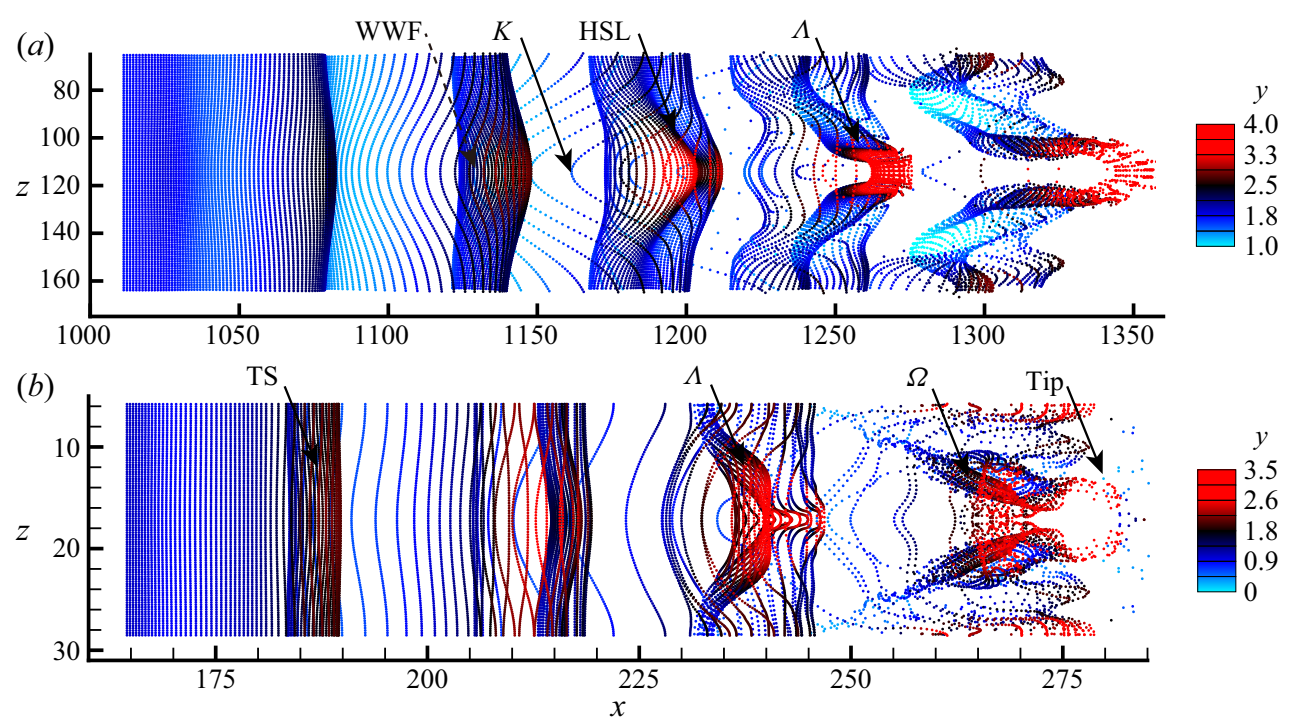

Figure 5. Timelines initiated at $y=1.71(\approx 24 \% \delta)$ for K-type transition with $(a)$ ZPG, and $(b)$ APG. Particles released at $x=1011.2$ and 164.5 for ZPG and APG, respectively.

the WWF is not observed, with the $\Lambda$-vortex appearing initially as a bow-shaped structure at $x \approx 235$, which seems to originate from a tilted transverse vortex tube upstream. Farther downstream, the $\Lambda$-vortex develops into an $\Omega$-shaped vortex, with its round tip moving rapidly away from the main body, creating a $\Lambda$-vortex with an open tip.

Figure 6 shows two stages of flow structure development for both the ZPG and APG cases illustrated by the deformation of a continuous timeline surface, similar to a continuous hydrogen bubble pattern obtained experimentally. The surface is displayed in greyscale, with the vertical location of the surface indicated by the scale to the right of the images. For the ZPG case in figure 6(a), it is observed that the surface appears to develop a new fold (labelled ' 2 nd $F$ '), which projects outward and above the portion of the surface marking the WWF (corresponds to the first fold, labelled ' 1 st $F$ '). This second fold corresponds to the HSL labelled in figure 5(a), where marked particles accumulate in a back-swept delta shape. Figure $6(b)$ shows that as time progresses, the second fold moves upward and develops into a $\Lambda$-shaped structure at $t=7.1 T$, corresponding to the $\Lambda$-vortex that was observed and labelled in figure $5(b)$. Figure $6(b)$ also shows the development of two streamwise vortices, labelled SV1 and SV2 near the legs of the $\Lambda$-vortices.

For K-regime transition with the APG, two stages of timeline surface development at $t=$ $3.2 T$ and $t=4.2 T$ are shown in figures $6(c)$ and $6(d)$, respectively. The tilted transverse vortex tube mentioned above is more clearly observed in figure 6(c), and is actually the second fold of the surface wrapped by the first fold of a WWF, similar to the N-regime transition in Jiang et al. (2020a). This second fold differs from that observed in the ZPG case, in that it does not project outward and above the surface of the WWF. By the $t=$ 4.2T stage, figure $6(d)$, the second fold develops into a $\Lambda$-shaped structure within the amplified and lifting WWF. The HSL for the APG case develops closer to the wall with a stronger transverse vorticity concentration $\left(\omega_{z}\right)$. This is hypothesized as the effect of the APG creating a more inflectional $U(y)$ profile, and precipitating a stronger inviscid instability. Above the $\Lambda$-vortex, the delta-shaped topology of the WWF is discernable. 

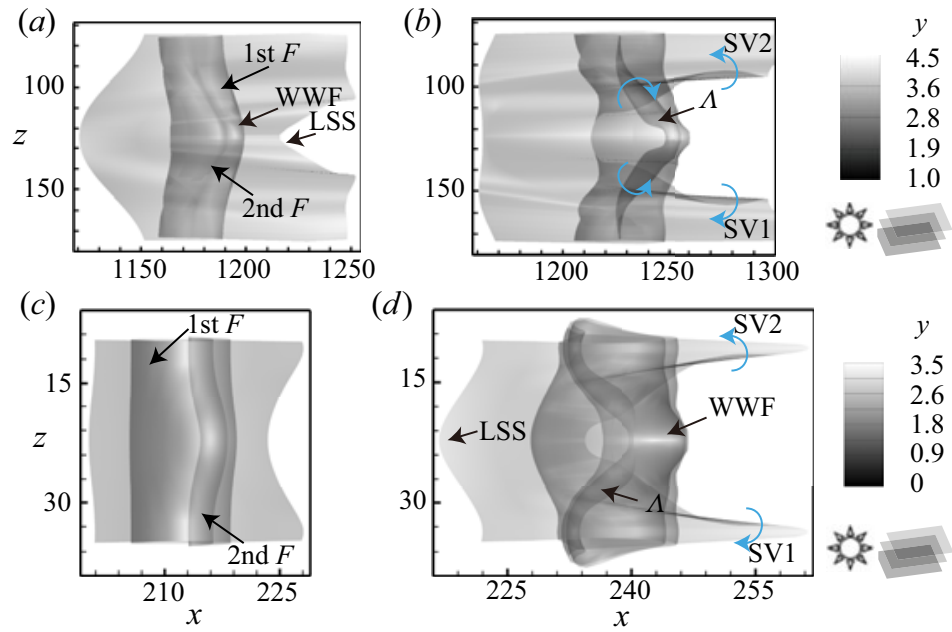

Figure 6. Evolution of timeline surfaces initiated at $y=1.71(\approx 24 \% \delta)$ for K-regime transition with ZPG at (a) $t=6.1 T$, (b) $t=7.1 T$, and for K-regime transition with APG at $(c) t=3.2 T$, (d) $t=4.2 T$. Here, $T$ is the period of corresponding primary waves, SV is a streamwise vortex and $F$ is a fold of the surface.
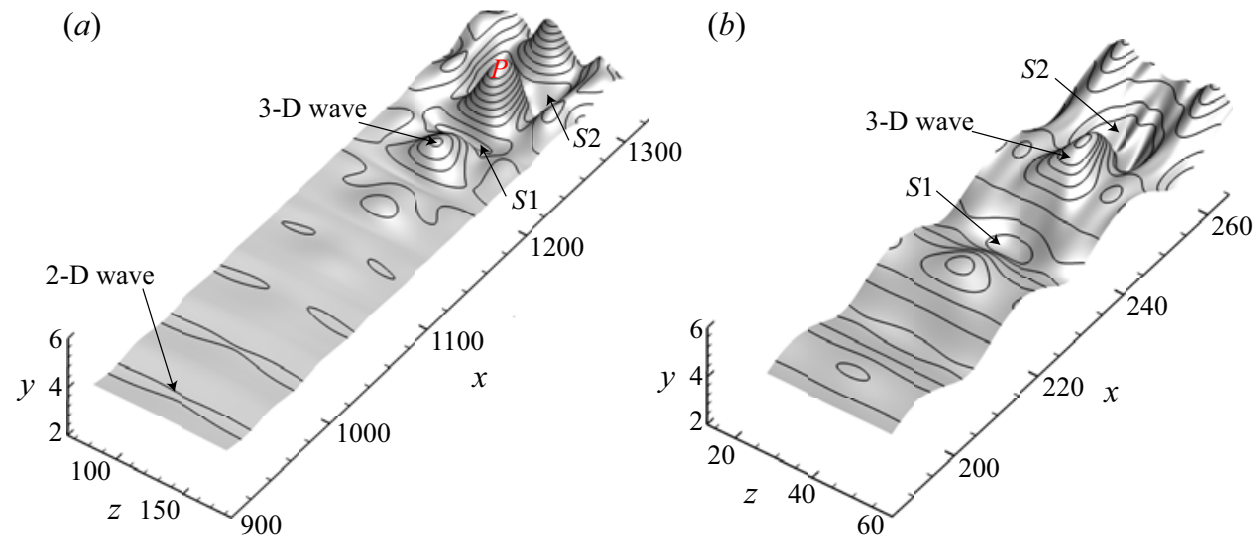

Figure 7. Material surface initiated at $y=3.83(\approx 53 \% \delta)$ for K-type transition at $t=T$ with $(a)$ ZPG (Jiang et al. 2020a) and (b) adverse pressure gradient.

Longitudinal rotation (labelled SV1 and SV2) and localized retardation (labelled LSS) of the timeline surface are similar for the two cases.

Figure 7 shows the deformation of an initially flat material surface initiated at $y=3.83$, for the two transition cases at $t=1 T$. The black contour lines illustrate respective changes in $y$ height, and show the development and growth of 3-D waves. In figure 7 $(a)$, the K-regime transition with zero pressure gradient, an upstream 2-D TS wave progressively amplifies into an apparent 3-D wave, appearing at approximately $x \approx 1200$. This 3-D wave subsequently amplifies with its wave front (labelled P) lifting-up, accompanied by regions of depression along and in front of the wave (labelled $S 1$ and $S 2$ ). For the K-regime transition with APG, shown in figure $7(b)$, the deformation of the material surface is similar to the case of the ZPG. As the 3-D wave develops downstream, a region of depression (labelled $S 1$ ) develops into a larger depression in front of the rising 3-D wave, 


\section{Three-dimensional wave structure in boundary layers}

labelled $S 2$. These depressions appear to be caused by the downward sweep of fluid from the outer layer due to the lift-up of the 3-D waves.

\section{2. $L C S$}

Understanding the behaviour of marked particles relies on the assessment of such properties as vorticity distributions or detection of coherent vortices. In the present study, LAE is applied to numerical and experimental flow data, to provide a simplified understanding of the overall flow geometry, which can help assess the flow physics creating the visualized patterns shown in $\S 3.1$.

\subsection{1. $L A E$}

In this work, the identification method used to extract vortical structures is based on the integral of enstrophy over a finite time interval of interest. We define the LAE as

$$
\operatorname{LAE}_{t_{0}}^{t_{1}}\left(\boldsymbol{x}_{0}\right):=\int_{t_{0}}^{t_{1}}\left|\boldsymbol{\omega}\left(\boldsymbol{x}\left(s ; \boldsymbol{x}_{0}\right), s\right)\right|^{2} \mathrm{~d} s,
$$

where $x_{0}$ is the initial position of a specific fluid volume, which is subsequently tracked from $t_{0}$ to $t_{1} ; \boldsymbol{x}\left(t, x_{0}\right)$ denotes the fluid trajectory starting at $x_{0}$ at time $t_{0} ; \omega$ is the vorticity along fluid trajectories defined as $\boldsymbol{\nabla} \times \boldsymbol{u}$ ( $\boldsymbol{u}$ denotes the velocity); and $|\boldsymbol{\omega}|^{2}$ is termed the enstrophy.

According to $\mathrm{Wu}, \mathrm{Ma} \& \mathrm{Zhou}$ (2006), the time evolution of the enstrophy is

$$
\frac{\mathrm{D}}{\mathrm{D} t}\left(\frac{1}{2} \omega^{2}\right)=-\boldsymbol{\omega} \cdot \boldsymbol{B} \cdot \boldsymbol{\omega}+\boldsymbol{\omega} \cdot(\boldsymbol{\nabla} \times \boldsymbol{a}),
$$

where $\boldsymbol{a}=\mathrm{D} \boldsymbol{u} / \mathrm{D} t$; and $\boldsymbol{B}$ is the surface deformation tensor. The first term of the right-hand side of (3.2) is the vortex stretching effect, which is a critical kinematic mechanism in the shearing process, and it is regarded as one of the most important keys to understanding vortical flows. Vortex stretching is responsible for the cascade process in turbulence, by which large-scale vortices become smaller and smaller with increasingly stronger enstrophy (Wu et al. 2006). The second term of the right-hand side of (3.2) contains the viscous effect. Thus, the LAE in (3.1) represents the combination of both inviscid stretching effects and viscous effects occurring between time $t_{0}$ and $t_{1}$.

\subsubsection{LAE-based structures in transitional flows}

Figure 8 shows 3-D structures and 2-D contours identified using the LAE method, based on the integral of the enstrophy (i.e. $|\omega|^{2}$ ) along fluid trajectories in K-regime transition with ZPG. The fluid domain $x_{0}$ is initiated at $1020<x<1270,75<z<175$ and $0<y<6$. To assure that the traced fluid domain $x\left(t, x_{0}\right)$ is within the geometry range of the PSE data sets, a tracking time interval of $\left[t_{0}, t_{1}\right]$ is set as $[0,0.34 T]$.

Three-dimensional isosurfaces of LAE are extracted $\left(\mathrm{LAE}_{0}^{0.34 T}=1.56 \mathrm{~s}^{-1}\right)$, as shown in figure $8(a)$. The surface colours reflect the respective wall-normal position. The surfaces clearly show the development from a 3 -D wave-like structure into a $\Lambda$-shaped structure. Prior to the appearance of a $\Lambda$-shaped structure, a tongue-shaped bulge appears (labelled $B$ ), which is analogous to the 'triangular bulge' observed in the transitional channel flow investigated by Zhao et al. (2016). Structure $B$ agrees with the back-swept HSL of the WWF in figure 5(a), which is responsible for the second fold behaviour of 


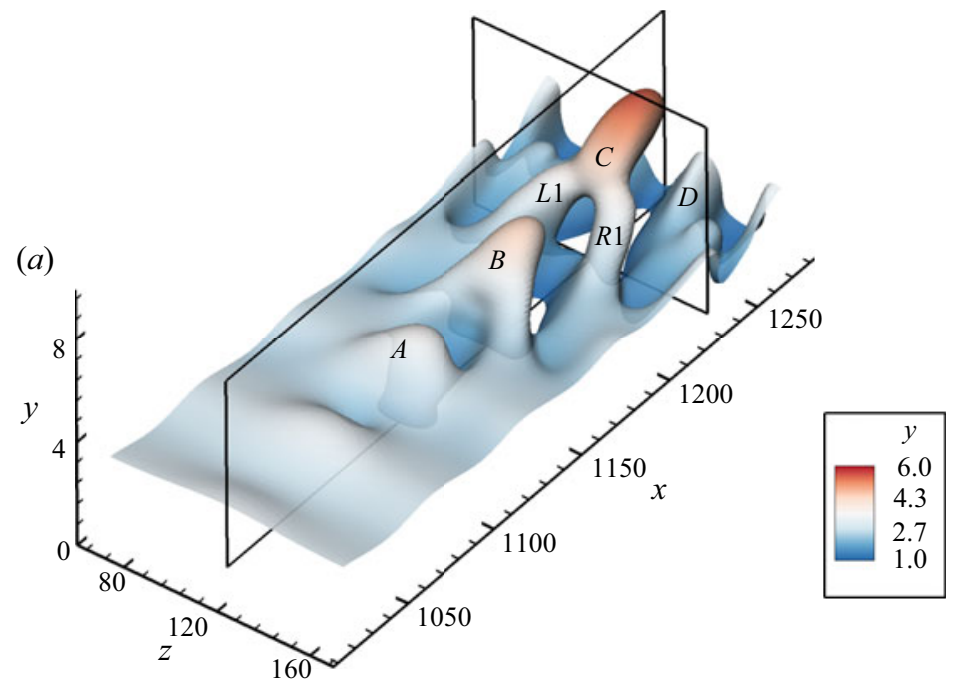

(b)

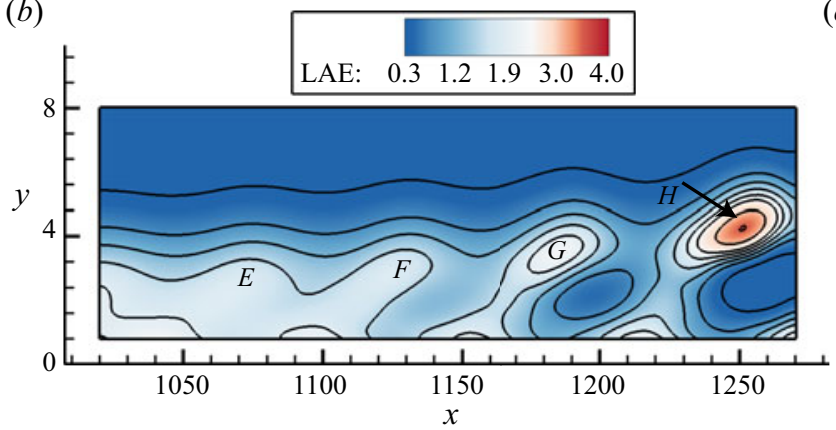

(c)

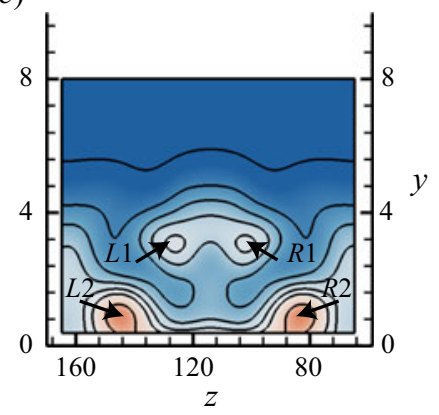

Figure 8. The LAE for K-type transition with a ZPG: (a) 3-D isosurfaces based on LAE detection $\left(\mathrm{LAE}_{0}^{0.34 T}=\right.$ 1.56 ), coloured by wall-normal position; (b) contours of LAE in $x-y$ plane at $z=114.7 ;(c)$ contours of LAE in $z-y$ plane at $x=1228$, using the same colourbar as panel $(b)$.

the timelines. Note that the tongue-shaped head (labelled $C$ ) of the $\Lambda$-vortex is different from a conventional one. This is because the LAE field for the boundary layer represents stretching caused by either a background field, or induced locally by the vortex itself. It is known that there already exists transverse vorticity $\left(\omega_{z}\right)$ in a boundary layer, which cannot be distinguished in (3.1) from the transverse vorticity created by a developing vortex within the boundary layer. Thus, an increased concentration of LAE near the head of a $\Lambda$-vortex will be reflected by the isosurface of LAE initially manifesting as a tongue-shaped structure.

The contours of LAE in an $x-y$ plane at $z=114.7$ (near the centreplane) and a $z-y$ cross-section at $x=1228$ are shown in figures $8(b)$ and $8(c)$. The strongest stretching is observed to occur at two locations: one is in the region labelled $H$ in figure $8(b)$, representing strong transverse stretching; the other is in two near-wall regions labelled $L 2$ and R2 in figure 8(c), representing strong streamwise stretching, which appears coincident with the regions labelled SV1 and SV2 in the timeline surface of figure 6(b). These patterns are considered to be quasi-streamwise vortices reflecting the legs of a previous hairpin (residing under the region labelled $D$ in figure $8 a$ ). As time progresses, these legs move toward the wall $(R 1 \rightarrow D)$, with their inclination angle decreasing, and the head of the $\Lambda$ vortex moving upward from the main body. The behaviour of the enstrophy 


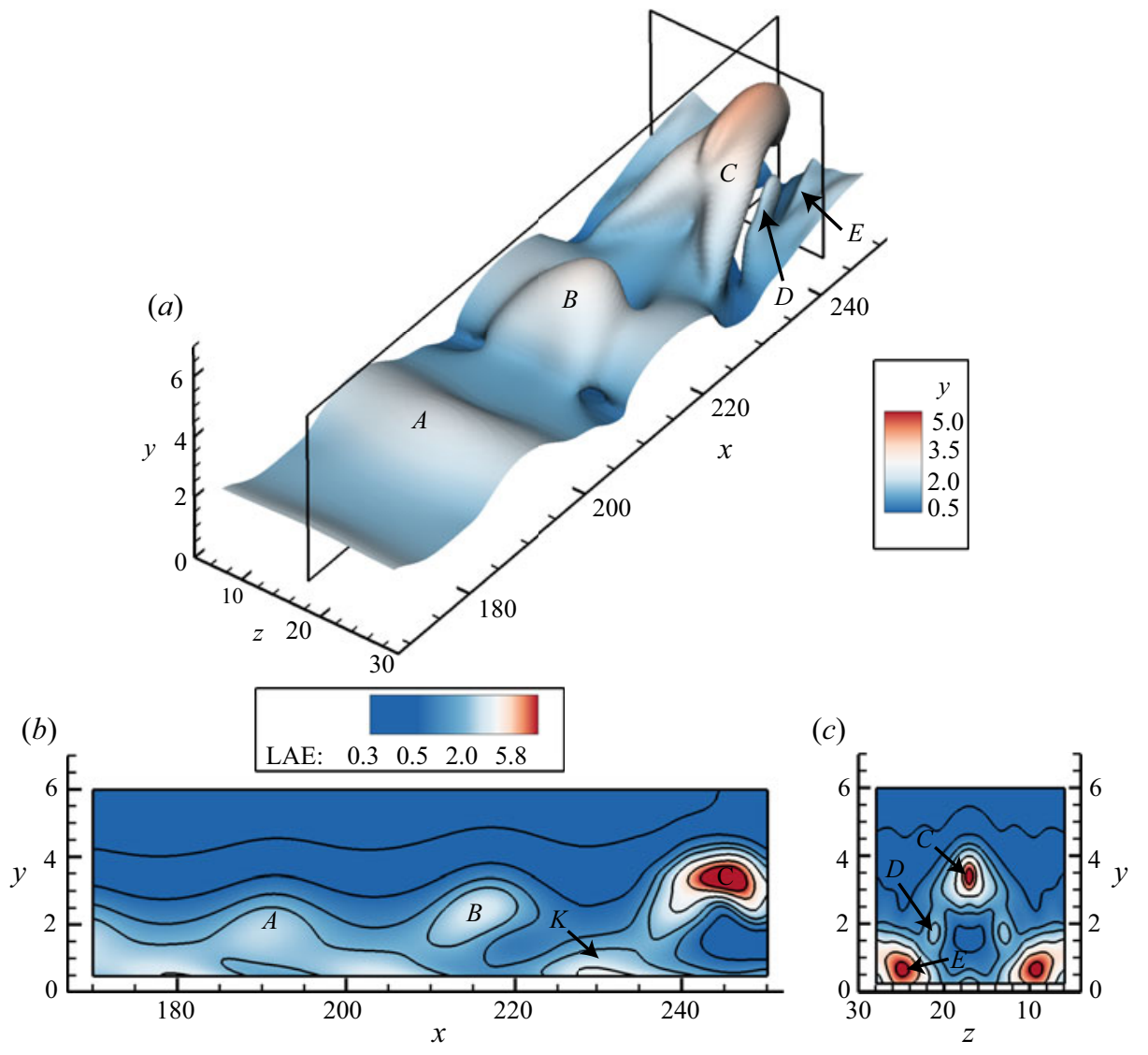

Figure 9. The LAE for K-type transition with an adverse pressure gradient: (a) 3-D isosurfaces of $\mathrm{LAE}_{0.3 T}^{0.7 T}=$ 1.8 , coloured by wall-normal position; $(b)$ contours of LAE in $x-y$ plane at $z=17 ;(c)$ contours of LAE in $z-y$ plane at $x=243$, using the same colourbar as panel $(b)$.

distributions from the regions $E-F$ to $G-H$ agrees with the wave-vortex development of marked particles, as illustrated in figure 5(a).

For the APG transition, 3-D and 2-D contours identified using the LAE method are shown in figure 9. The fluid domain $x_{0}$ was initiated at $170<x<250,12<z<36$ and $0<y<6$, focusing on the stage where the $\Lambda$-vortex develops from a 3-D wave structure. The time interval was set as $[0.3 T, 0.7 T]$ to assure the domain is within the geometry range of the data set during the period.

Figure $9(a)$ shows the isosurface of $\mathrm{LAE}_{0.3 T}^{0.7 T}=1.8$, again coloured by wall-normal position. Compared with the ZPG case, the structure development from a 3-D wave to a $\Lambda$-vortex is more rapid for the APG, with the $\Lambda$-vortex appearing to be inclined at a steeper angle. It is worth noting that the isosurface of LAE is not the boundary of vortices, but reflects the trajectory of the vorticity concentration over a specified period. The deformation of the isosurface appears coincident with the deformation of the timeline patterns (WWF $\rightarrow \Lambda$-vortex) in figure $5(b)$. The warped head of a $\Lambda$-vortex is observed in the $C$ region, which is different from the of ZPG case. Jiang et al. (2020a) proposed that the vorticity concentration and HSL at the border of the WWF are the result of a lifting 3 -D wave, which further develops into a $\Lambda$-vortex. The visualized structures may represent 
X.Y. Jiang, D.W. Gu, C.B. Lee, C.R. Smith and P.F. Linden

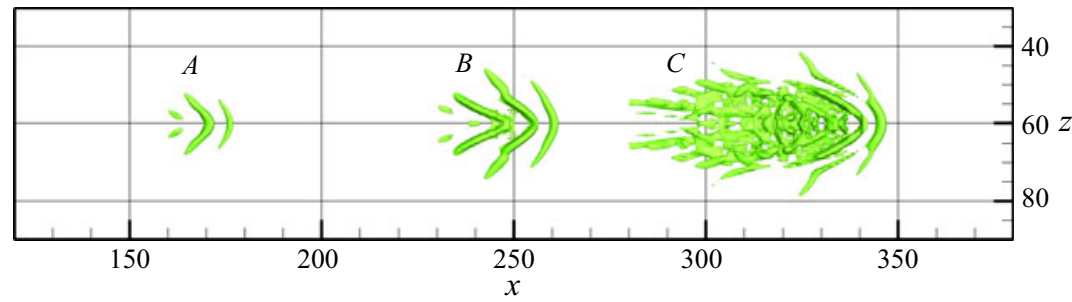

Figure 10. Turbulent spot development using isosurfaces of $\lambda_{2}$ criteria at non-dimensional times $t=180,270,360$ at Ma 3(from left to right), $\lambda_{2}=-0.001$.

Lagrangian-averaged shear layers developing in front of the WWF, where high vorticity concentrations are present.

The feature of a warped head is also clearly shown in figure $9(b)$, which shows a stronger transverse shear layer in front of the head region, leading to a downward sweep of the outer flow. The multiple folding process of the timelines in figure 6 and the large depression $(S 2)$ in figure $7(b)$ can be explained by this enstrophy concentration. In the near-wall region, another concentration of LAE is observed, labelled K in figure $9(b)$, indicating high-shear layers evolving close to the wall at this location. The observation of the lower vortices agrees well with the study by Kloker \& Fasel (1995), who pointed out the existence of a 'lower inverted HSL' in a decelerated flow with $\beta_{H}=-0.18$.

Figure $9(c)$ shows contours of LAE in an end-view $z-y$ section at $x=243$, which shows the presence of a pair of primary vortices near the wall (one is labelled $E$ ), as well as small vortices above them (one is labelled $D$ ). The distribution of these vortices is similar to those shown in figure $8(c)$ for the ZPG case. There is also a concentration of LAE in the middle region, in accordance with the vortex head labelled $C$, indicating a stronger spanwise and streamwise stretching process occurring for the APG case, as compared with the ZPG case.

\section{Turbulent spot in bypass transition}

\subsection{Development of the turbulent spot}

The DNS was performed on a flat plate at Ma 3. A turbulent spot was generated by introducing a localized disturbance on the wall surface near the leading edge. Figure 10 shows the development of a turbulent spot from a linear wave packet until nonlinear breakdown. Time has been normalized by length $1 \mathrm{~mm}$ and free stream velocity. At $t=180$, the wave packet transports within a linear region, which is initiated by a small point disturbance. Due to instability of the boundary layer, the wave packet becomes 3-D (labelled $A$ ). The first-mode wave transports fastest as an oblique wave, and the second-mode wave is not important in this case. By $t=270$, the wave packet has travelled farther downstream, is of a larger amplitude (labelled $B$ ), undergoes a nonlinear growth, and is close to breakdown. The wave packet propagates in both lateral and streamwise directions. As the wave packet becomes more unstable, the rear wave grows more 3-D, like a $\Lambda$ structure (see the stage at $x \approx 250$ ). By $t=360$, the wave packet amplitude has grown to a point of unstable breakdown (labelled $C$ ). Following the breakdown, a turbulent spot develops as an arrowhead-shaped structure. The shape of the spot is consistent with the typical structure described by Krishnan \& Sandham (2006) and others. 
(a)

$$
\begin{array}{lllllll}
y & 1.7 & 1.8 & 2.0 & 2.1 & 2.2
\end{array}
$$

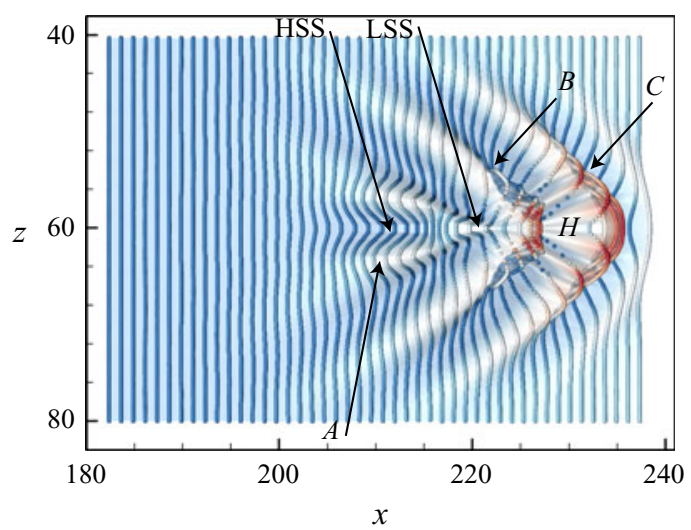

(b)

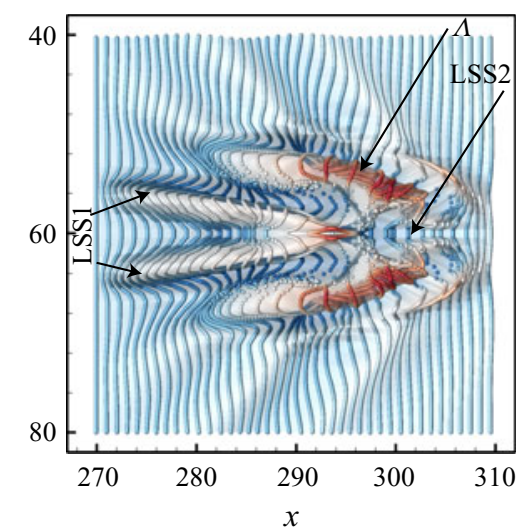

Figure 11. Timelines of wave packet development within the linear and nonlinear regions: $(a)$ timelines initiated within the linear region at $x=182,(b)$ timelines initiated within the nonlinear region at $x=270$. (See the supplementary movies 1 and 2 for the complete evolution.)

\subsection{Timelines and material surfaces}

To examine the details of the spot development, timelines are initiated in $x=182$ and $x=$ 270 to illustrate the timeline patterns within the linear and nonlinear regions. Figure 11 $(a)$ shows the evolution of the wave packet within an early linear region (see supplementary movie 1 available at https://doi.org/10.1017/jfm.2020.1023 for the complete evolution). The colour of the points indicates the wall-normal distance. There are two wave fold structures (labelled $B$ and $C$ ), with an HSL forming between the two waves. As a result, fewer particles are present in this position, e.g. at the centre of the wave packet $z=60$, a 'bubble-free heart-shaped' region (labelled $H$ ) appears at $x=220-235$ due to the HSL. The 'heart-shaped' region caused by a sequential wave folds is analogous to the hydrogen bubble visualizations shown in Acarlar \& Smith (1987a) that are caused by the induced flow of two sequential hairpin heads. In the region between $x=202-214$, timelines show a high-speed streak (HSS) developing on the centreline, accompanied by two tilted LSS to either side of the centreline. The HSS just follows an LSS caused by the wave fold $B$, which creates a squeezing effect at position $x=216$. Another wave fold is generated at this location, which causes the wave packet to grow in the streamwise direction.

The nonlinear development of the wave packet is shown in figure 11(b), which is close to the breakdown region. Here, the timelines are initiated at $x=270$ and $z=42-74$. An apparent $\Lambda$-vortex is observed in the middle of the packet, which causes particles to roll up near the location of the hairpin legs. Two LSS appear at the rear of the wave packet, which looks markedly similar to conventional LSS observed in a turbulent boundary layer. However, these LSS are clearly observed to develop from a weak wave structure (region labelled $A$ in figure 11a), and not from the presence of vortices. The particles between the two wave folds move toward the edges between the LSS and HSS, where an HSL develops (see supplementary movie 2 for the complete evolution).

Figure 12 shows the evolution of material surfaces in proximity to the spot at different wall-normal distances, i.e. $y=2.4$ and $y=0.98$. The contour colours (with contour lines) show the distance from the wall surface. Within the upper material surface at $y=2.4$, the influence of the spot appears as a pair of oblique waves due to the 3-D instability. 

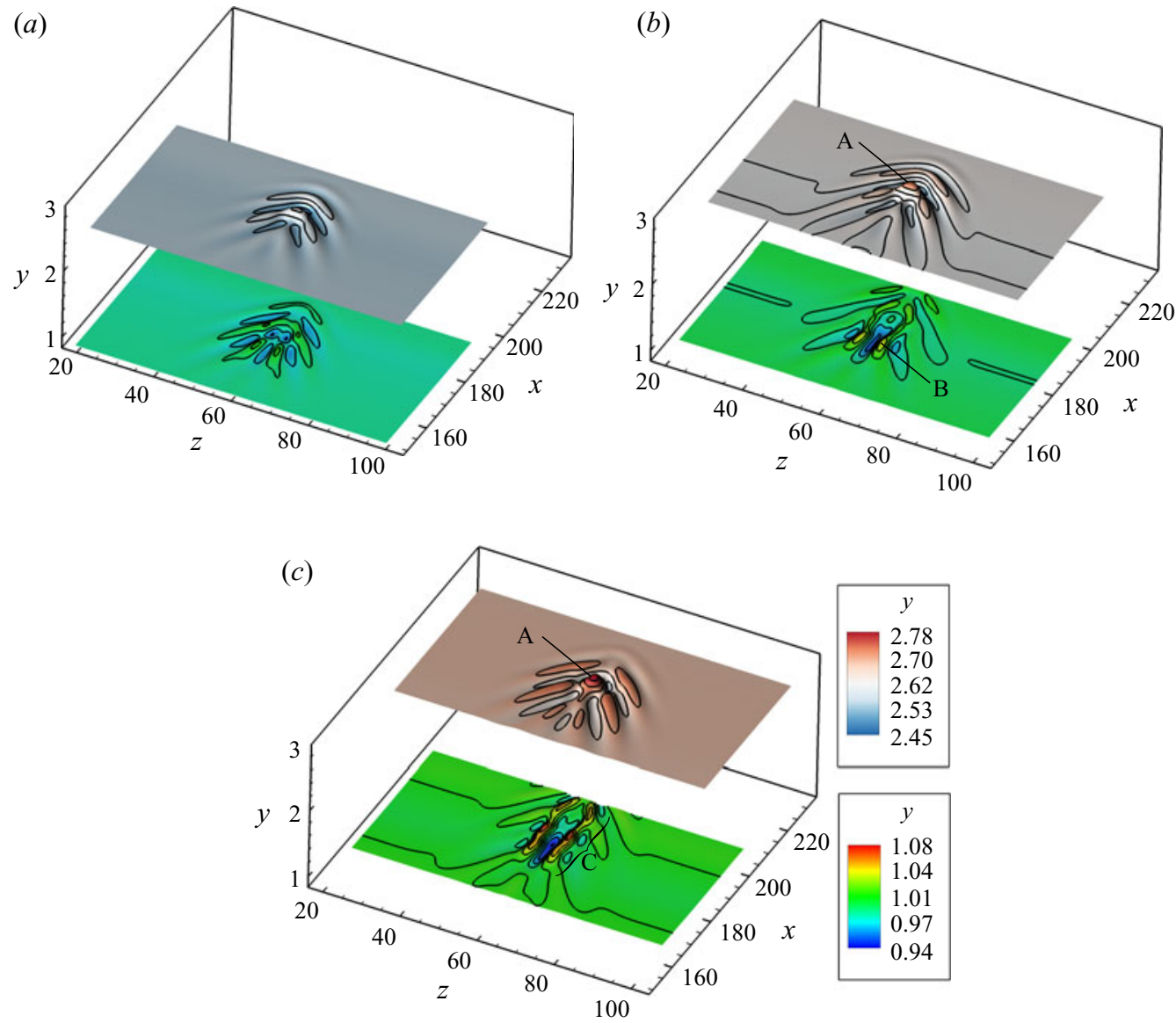

Figure 12. The evolution of material surfaces initiated at $y=0.98$ and 2.5: (a) $t=194$; (b) $t=209$; (c) $t=224$. (See the supplementary movie 3 for the complete evolution.)

During the development, an apparent 3-D wave appears within the middle of the packet (labelled $A$ ). For the material surface initiated at $y=0.98,3-\mathrm{D}$ waves also form close to the wall, labelled as $B$ in figure $12(b)$. By $t=224$, figure $12(c)$ shows that for the material sheet at $y=0.98$ three 3 -D waves align into a streamwise pattern, which has the appearance of an LSS. This pattern is similar to the O-regime transition in Jiang et al. (2020a).

\subsection{LAE contours within the turbulent spot}

Figure 13 shows the LAE for the turbulent spot, integrated from time $t=180$ to $t=$ 270. Figure 13(a) shows an isosurface of $L A E_{180}^{270}=20 \mathrm{~s}^{-1}$, coloured with wall-normal distance. The upper layer at $y \approx 1.8$ reveals a pattern of oblique waves, which is consistent with the upper material surface pattern of figure 12. Figure 13(b) shows an isosurface of $L A E_{180}^{270}=26 \mathrm{~s}^{-1}$, which appears as three parts. The first part (labelled $A$ ) is a near-wall structure. This part distributes laterally due to the transverse vorticity which is large near the wall surface. However, the head of structure $A$ shows a streamwise concentration of enstrophy, which is associated with LSS within the near-wall region, whose presence were shown previously in figure $12(c)$. The second part (labelled $B$ ) is a $\Lambda$-shaped structure, which is at a wall-normal distance $y \approx 1.5$. The nearby fluid particles rotate around the 


\section{Three-dimensional wave structure in boundary layers}

(a)

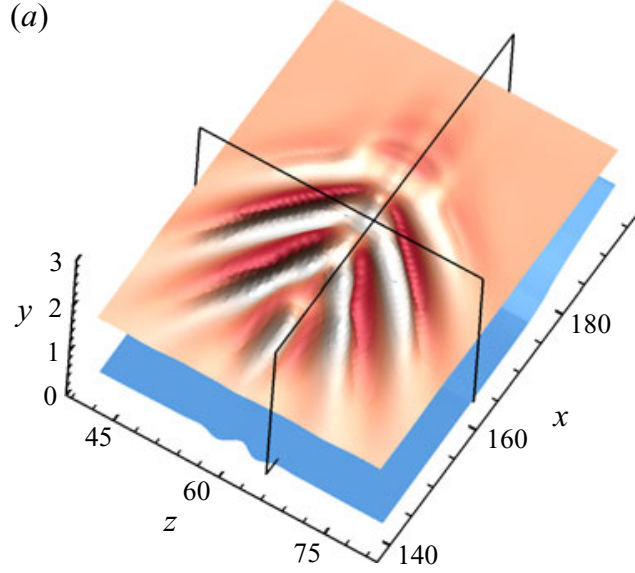

(c)

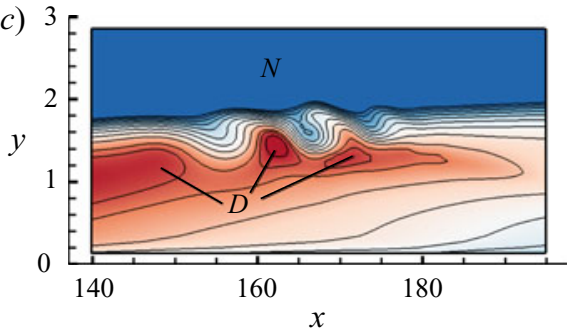

(b)
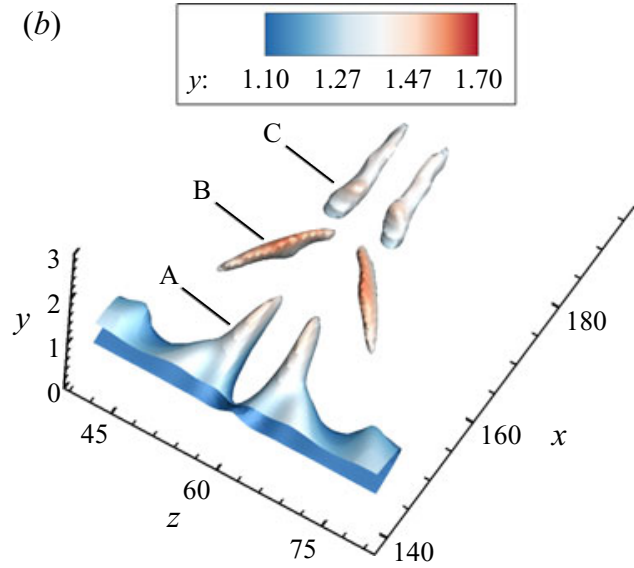

(d)

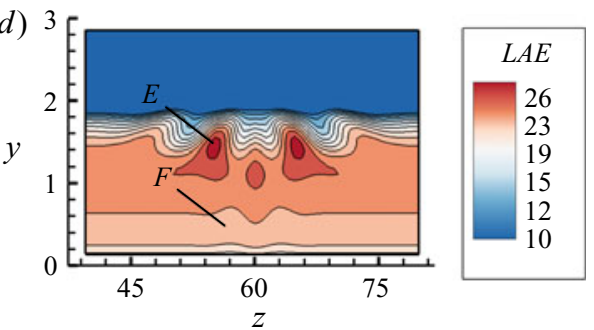

Figure 13. The LAE ( $\operatorname{LAE}_{180}^{270}$ ) for a turbulent spot: (a) 3-D isosurfaces of LAE (LAE $=20$ ), coloured with wall-normal position; (b) 3-D isosurfaces of LAE (LAE $=26$ ); (c) contours of LAE in $x-y$ plane at $z=65$; $(d)$ contours of LAE in $z-y$ plane at $x=163$. The same colourbar applies for panels $(c)$ and $(d)$.

centreline of the legs of a $\Lambda$-vortex, which agrees with the behaviours of timeline particles in figure 11. The third part (labelled $C$ ) shows two streamwise vortices in front of the $\Lambda$-shaped structure, with the mechanism for their formation being unclear.

Figure $13(c)$ shows the contours of LAE in the $x-y$ plane at $z=65$, as there is no special structure on the centreline $z=60$. The main LAE contours concentrate at $y \approx 1.0 \sim 1.5$ (labelled $D$ ), which is consistent with the $\Lambda$-vortex structure shown by figure $13(b)$. The contour lines are weakly inclined at a small angle in the streamwise direction, which means the $\Lambda$-vortex has a weak lift-up influence on the nearby fluid particles. An apparent interface is observed at $y \approx 1.7$, appearing as a wave-like undulation. This interface separates the strong mixing zone $(D)$ from the non-mixing zone $(\mathrm{N})$. Figure $13(d)$ shows the LAE contours in the $z-y$ plane at $x=163$. The structure labelled $E$ is one leg of a $\Lambda$-vortex, while the hump structure near the wall surface (labelled $F$ ) is an LSS formed by 3-D waves, as was also shown by figure $12(c)$.

\section{Turbulent boundary layer}

\subsection{Description of data set}

The experimental data set used in this study was obtained using tomographic particle image velocimetry (Tomo-PIV), which is the same as Jiang et al. (2020b). A brief introduction of the experiment is given here. The experiment was performed in the Peking University water tunnel (PUWT), which is an open-surface recirculating water channel with a cross-section of $0.4 \mathrm{~m} \times 0.4 \mathrm{~m}$ and length of $6 \mathrm{~m}$. The tunnel free stream velocity 


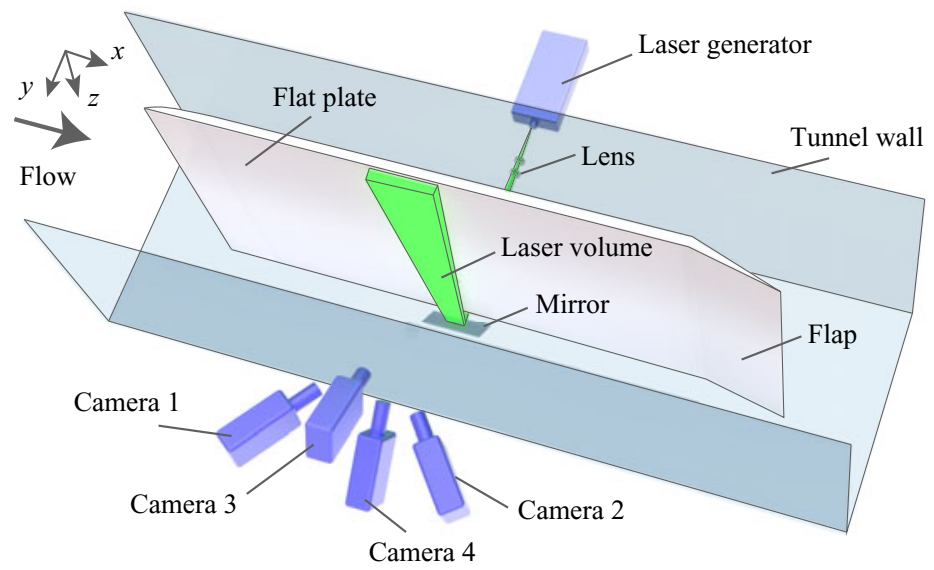

Figure 14. Experimental set-up for Tomo-PIV (Jiang et al. 2020b).

range is from 0.1 to $1.3 \mathrm{~m} \mathrm{~s}^{-1}$, with a turbulence level of $0.77 \%$ for tunnel velocities of 0.1 to $0.3 \mathrm{~m} \mathrm{~s}^{-1}$. The plate has a chord length of $1.8 \mathrm{~m}$, a span of $0.8 \mathrm{~m}$ and a thickness of $15 \mathrm{~mm}$. The flat plate was mounted vertically on the centreline of the tunnel at zero angle of attack. A trailing flap was mounted at the end of the plate to introduce circulation and thus adjust the stagnation point on the leading edge. There would be a separation bubble at the tip of leading edge without using a flap. Adjusting the trailing flap to a suitable angle caused early boundary layer transition to turbulence at $x \approx 400 \mathrm{~mm}$. This is similar to using a trip wire to initiate transition. Hydrogen-bubble timelines visualization was used to monitor the flow state during the adjustment process, starting from a laminar flow. The angle of the flap was gradually reduced until intermittent flows with random LSS began to appear in the hydrogen bubble pattern.

The free stream velocity employed for Tomo-PIV was approximately $0.17 \mathrm{~m} \mathrm{~s}^{-1}$, and the distance, $x_{d}$, from leading edge to the centre of the measurement region was $502 \mathrm{~mm}$. The Tomo-PIV configuration is shown in figure 14. Four high-speed cameras (Photron FASTCAM SA4) with a resolution of $1024 \times 1024$ pixels were used, in a cross-configuration arrangement. The cameras are fitted with Nikon lenses of $200 \mathrm{~mm}$ focal length. The Tomo-PIV sampling frequency was $500 \mathrm{~Hz}$, i.e. time increments $\Delta t^{+} \approx$ 0.42 viscous time units. Illumination was provided by a high-speed laser generator from Beijing ZK Laser, DCQ-30Q, a single-cavity double-pulse laser system with a beam wavelength of $527 \mathrm{~nm}$. The four camera/laser system was synchronized using a LaVision's PTU timing controller. The mean diameter of the tracing particles is $11 \mu \mathrm{m}$. Experiments were conducted at a particle image density of 0.098 particles per pixel.

Volume self-calibration (Wieneke 2008) was used on the preprocessed particle images. Volume reconstruction was achieved by sequential motion tracking enhancement (known as SMTE) (Lynch \& Scarano 2015). The reconstructed volume was $1041 \times 1048 \times 187$ voxels. A multipass approach of four steps was carried out for the volume correlation, and the interrogation volume size for the final pass was $16 \times 16 \times 16$ voxels with a $75 \%$ overlap. The spatial resolution was 12.52 wall units, with grid spacing of 3.13 wall units. As a final step, a Gaussian smoothing filter of $3 \times 3 \times 3$ subvolume ( 2.3 wall units) was applied to the velocity data. Proper orthogonal decomposition analysis was finally applied to the smoothed velocity data sets, and the first four leading-order proper orthogonal 

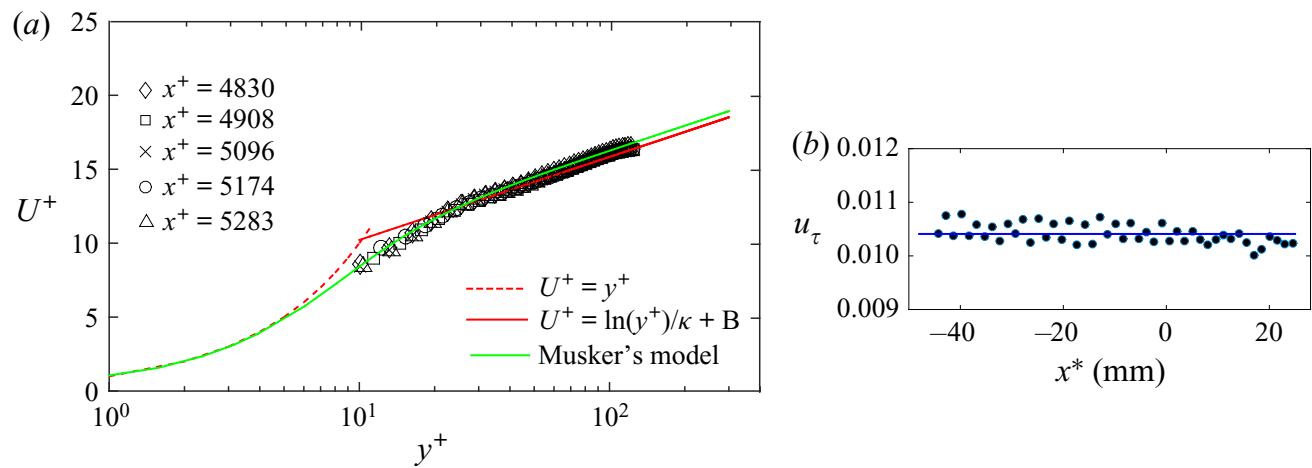

Figure 15. (a) Turbulent boundary layer mean velocity profile plotted on log-linear coordinates (Jiang et al. $2020 b$ ). Fitting curves using model of Musker (1979): $\kappa=0.41 ; B=5.0$. (b) Variation of friction velocity calculated by fitting the mean velocity profile. Here $x^{*}$ is the physical streamwise distance relative to the calibration centre of the Tomo-PIV measurement.

decomposition modes are extracted for noise removal and the extraction of higher modes (Wu 2014; Deng et al. 2018).

Based on 3400 frames of velocity field obtained from the Tomo-PIV data at a sampling rate of $500 \mathrm{~Hz}$, the mean turbulent boundary layer velocity profile obtained from the Tomo-PIV data is shown in figure 15, compared with a universally fitted curve for turbulent boundary layers according to the model of Musker (1979). The friction velocity is obtained by fitting the curve of Musker (1979) to the experimental data. An iterative optimization procedure based on a gradient descent algorithm was used for the fitting process. The result gives an average of $u_{\tau}=0.0104 \mathrm{~m} \mathrm{~s}^{-1}$, with a root mean square variation of $1.77 \times 10^{-4}$, as shown in figure $15(b)$. The average friction velocity was used to normalize the coordinate and time parameters in this study.

\subsection{Flow visualization}

Figure 16 shows plan-view timeline patterns for LSS. The LSS were identified by tracing the peak of the timelines with a narrow or elongated retardation region, as was identified in the literature (Kline et al. 1967; Kim, Kline \& Reynolds 1971; Smith \& Metzler 1983). The plan-view timelines were generated at $y^{+}=27$. The figure shows that the timelines, which flank the low-speed region, appear to temporally intersect and crimp, as shown in the regions labelled $A$ and $B$ in figure 16, which appear similar to the pattern labelled LSS1 in figure 11(b). Figure 16 also illustrates two phenomena: (i) the locations of the LSS are adjacent to the locations of higher wall-normal position; and (ii) the flank of the LSS is usually the most unstable region that initiates the breakdown of the LSS pattern, by attracting or repelling neighbour particles.

In order to further assess 3-D behaviour of an LSS in a turbulent boundary layer and its relationship to a 3-D wave and hairpin vortices, the LSS in figure 16 was further examined. Figure 17 is an oblique view of the development of vertical material sheets initiated in the $z-y$ plane at $x^{+}=-416$ from $t^{+}=0-32$ at time intervals of $t^{+}=8$. These 3-D representations of the material sheet evolution (within a cross-section) show clearly that near-wall fluid moves upward (labelled L) in a region of an LSS, flanked by the movement of outer region fluid downward at the sides of the streak (labelled S), as indicated by the yellow arrows. As shown in figure 17, as time progresses the fluid in the LSS regions 


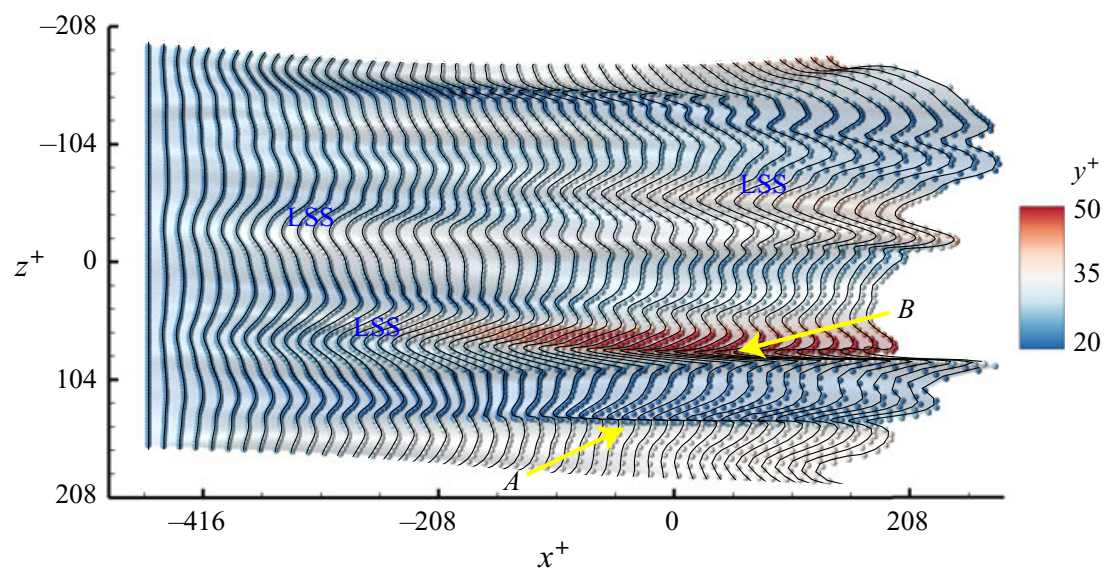

Figure 16. Timelines initiated at $y^{+}=27$ at $t^{+}=0$. The colour contours indicate the wall-normal position of the timeline surface.

(e.g. regions labelled $L 1, L 2, L 3$ ) develops into bulge structures at a downstream position (labelled $A, B, C$ ), creating apparent inflectional profiles, which is analogous to the typical bursting behaviour observed by Kim et al. (1971). The inflectional region moves away from the wall, creating the same type of kinking behaviour observed in the hydrogen bubble visualizations of Hama \& Nutant (1963). According to simulations of early (Jiang et al. 2020a) and late (Zhao et al. 2016) transitional flow, a bulge is present before the development of hairpin-like structures, which indicates the bulges may be 3-D structures within the boundary layer. In the region labelled $C$ in figure 17 (corresponding to the $A$ region in figure 16), a bulge appears to rotate at the flanking boundaries (labelled $R$ ) and begins to roll up into what is interpreted as counter-rotating streamwise vortices.

The evolution of $x-z$ material surfaces, initiated at a series of $y$ locations, and spanning the region $-133<z^{+}<74$ where LSS appear, is shown in figure 18. As illustrated, these surfaces undergo significant transverse warping, revealing substantial spanwise variations. The evolution of the 'peak-valley' patterns reflected by the deformation of the initially planar material surfaces shows strong movement upward, away from the wall, revealing a lift-up behaviour (labelled $L 1$ and $L 2$ ), which is consistent with the behaviour of the vertical material surfaces shown in figure 17. The lift-up behaviour initiates near the wall, and progressively develops a 3-D wave-like pattern. Note that two new kinks appear to the sides of the two main peaks within the near-wall region (indicated by arrows labelled L3 and L4 on figure 18c), which destabilize the LSS, indicating the flank of LSS is most unstable.

\subsection{LAE contours within the turbulent boundary layer}

Figure 19 shows LAE contours in the $x-z$ and $z-y$ planes, and the identified Lagrangian vortex boundaries via isosurfaces of LAE, in the region adjacent to the location of LSS. Two integral intervals with the same time span were applied in computing LAE, i.e. $t^{+}=[0,40]$ and $t^{+}=[10,50]$. Figures $19(a)$ and $19(b)$ are contours of $\operatorname{LAE}_{0}^{40}$ at $y^{+}=17$ and $x^{+}=104$, respectively, with isosurface of $\mathrm{LAE}_{0}^{40}=4500 \mathrm{~s}^{-1}$ being superimposed on figure $19(a)$. Two apparent vortices are detected at $z^{+} \approx 65$ and $z^{+} \approx 100$, which are located to either side of the $L 2$ of figure 18. The vortex core to the right (marked $B$ in figure 19a) is almost twice the LAE value as that of vortex core $A$. This means that the 


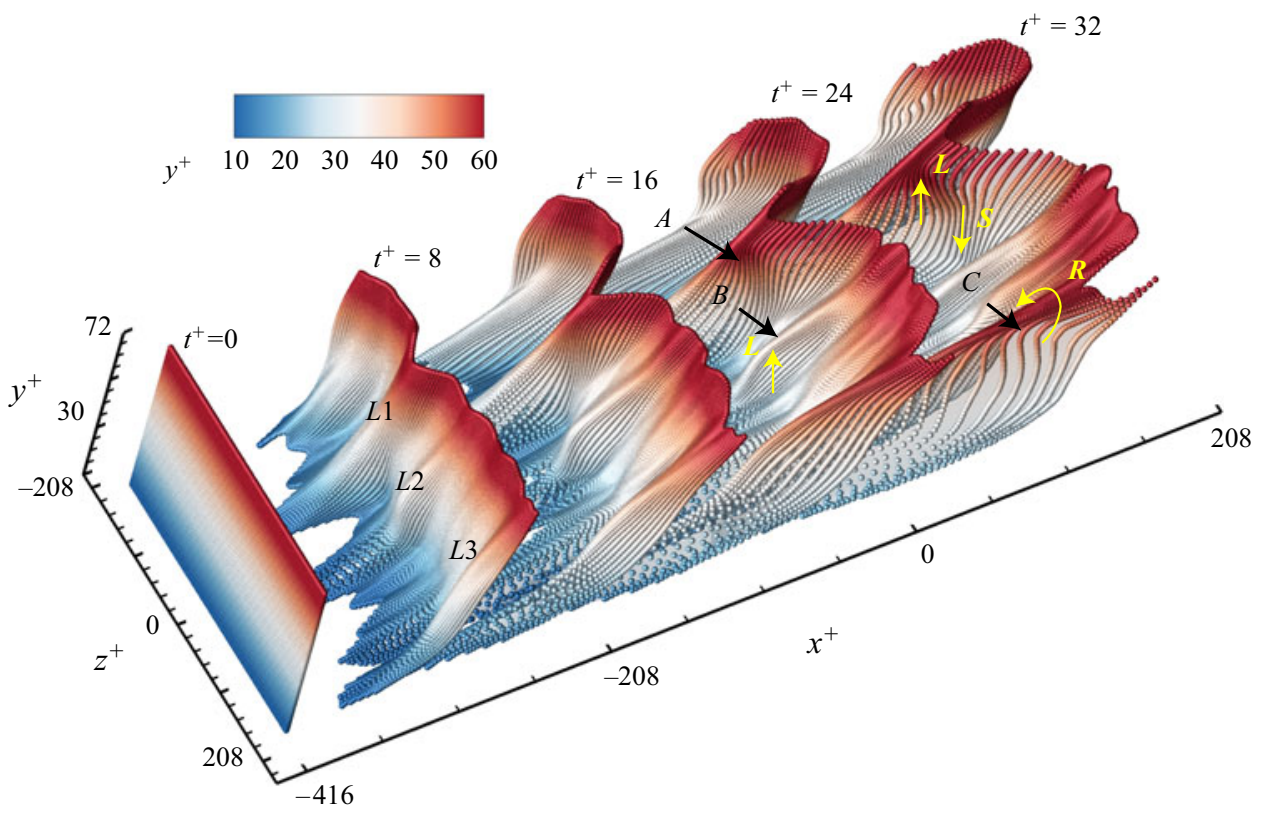

Figure 17. Temporal evolution of vertical material sheets in $z-y$ plane initiated at $x^{+}=-416$ from $t^{+}=0-32$. Vertical material sheets in the $z-y$ plane are released at time intervals of $t^{+}=8$.
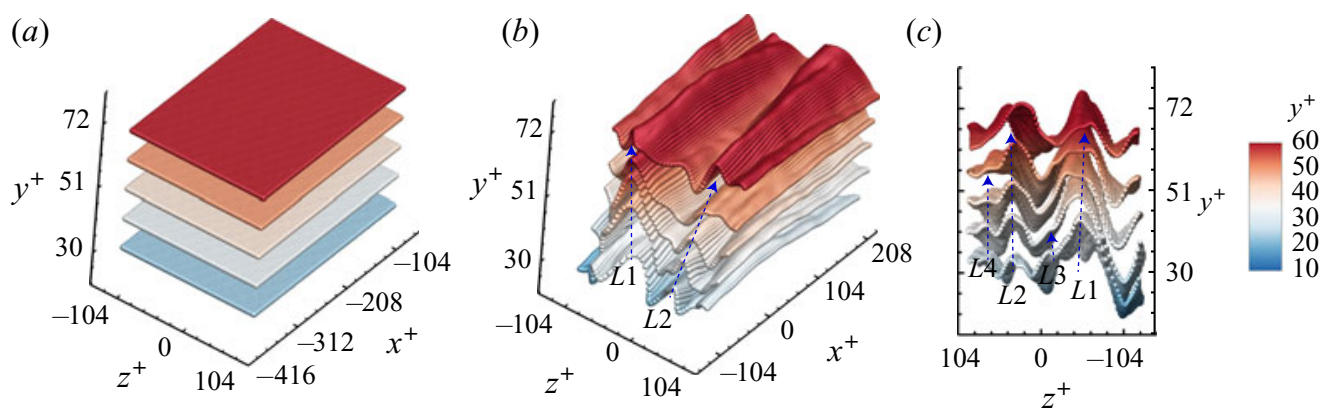

Figure 18. Evolution of material surface initiated at $y^{+}=22,32,42,52$ and $62:(a) t^{+}=0 ;(b) t^{+}=20$; (c) end-view of panel $(b)$. The initial range of the surfaces: $-133<z^{+}<74$; $-372<x^{+}<-74$.

vorticity is more concentrated on the right-hand side of the streak. Note that vorticity concentrations in regions labelled $C$ and $D$ in figure $19(b)$ are considered to be weak, which is closely related to the lift-up of streak $L 1$ of figure $18(c)$.

Figures $19(c)$ and $19(d)$ show the contours of $\operatorname{LAE}_{10}^{50}$ for the same planes as figures $19(a)$ and $19(b)$, taken $\Delta t^{+}=10$ after figures $19(a)$ and $19(b)$. The streamwise structures shown in figure $19(c)$ are isosurfaces of $\mathrm{LAE}_{10}^{50}=4500 \mathrm{~s}^{-1}$, which evolve from the main structures shown in figure 19(a), though with some deviation from their previous locations. A comparison of figures 19(a) and 19(c) suggests a persistence and dominance of the right vortex $B$ after $\Delta t^{+}=10$, which may explain the new lift-up of $L 4$ in figure $18(c)$ and the rotation $C$ in figure 17. Two LAE concentration regions in figure $19(c)$, labelled $C$ and $D$ at $z^{+} \approx-156$ and $z^{+} \approx-83$, seem to develop from the weaker regions within the dashed 
(a)

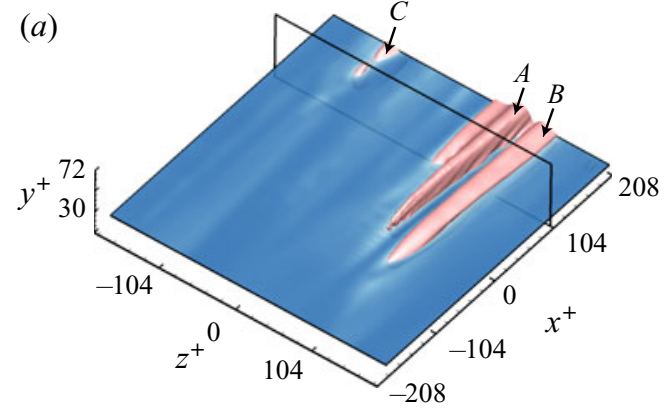

(b)
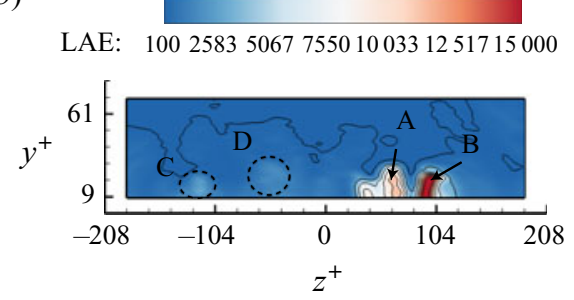

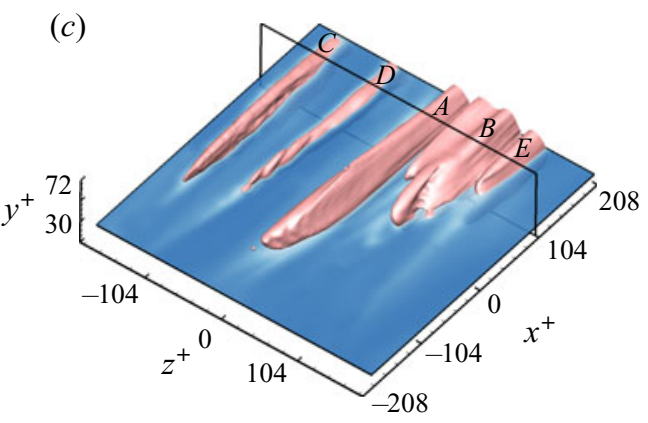

(d)

LAE: 100258350677550100331251715000

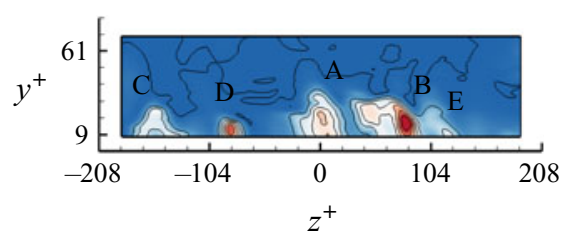

Figure 19. Contour of LAE in a turbulent boundary layer: (a) contours of $\operatorname{LAE}_{0}^{40}$ at $y^{+}=17$, superimposed with an isosurface of $\mathrm{LAE}_{0}^{40}=4500 \mathrm{~s}^{-1} ;(b)$ end-view contours of $\mathrm{LAE}_{0}^{40}$ at $x=104 ;(c)$ contours of $\mathrm{LAE}_{10}^{50}$ at $y^{+}=17$, superimposed with an isosurface of $\operatorname{LAE}_{0}^{40}=4500 \mathrm{~s}^{-1} ;(d)$ end-view contours of $L A E_{10}^{50}$ at $x^{+}=104$.

circles in figure $19(b)$. Note that regions $D$ and $E$ develop on the left and right flanks of the LSS labelled $L 1$ in figures 17 and 18.

The 3-D evaluation of the tomographic timelines demonstrates that the LSS is closely associated with 3-D wave behaviour (figure 16), which is similar to the pattern shown for the transitional boundary layer (figure 5) and bypass transition (figure 11). The spatio-temporal deformation of an advected material surface (figure 18) also reveals a 3-D wave behaviour of the LSS, with the subsequent breakdown resulting in ejections and sweeps associated with the bursting process. The quasi-streamwise vortices detected using 3-D LAE criteria shown in figure 19, are identified as the roll up occurring at the high shear interface of 3-D waves on a streak, which are hypothesized to be generated by the amplification and breakdown of the 3-D wave behaviour of the LSS.

\section{Discussion}

The presence of an APG causes the flow structure to develop more rapidly than for a ZPG case. It is well known that bursting activity increases in an APG turbulent flow, and the advection of hairpin vortices (particularly the legs) relative to the wall is decelerated by an APG (Kline et al. 1967; Smith et al. 1991). However, it is difficult to determine a quantitative relationship for the differences and similarities between flows of an APG and a ZPG. Based on the results of the LAE, an APG promotes the development of 3-D waves, enhances the vorticity concentration near the boundary of the waves, which facilitates the breakdown of the transitional flows. As is mentioned in $\S 3.2$, LAE represents the averaged effect of both inviscid stretching effects and viscous effects during the time of interest. Viscous effects are the most important in the inner regions near the wall, having a significant role in dissipation. The trailing legs of the hairpin vortex move progressively down toward the surface where they will either dissipate (for weak legs), or to provoke an 


\section{Three-dimensional wave structure in boundary layers}

eruption of fluid and/or vorticity away from the wall (for strong legs). However, above the inner viscous layer, flow evolution is primarily inviscid, with wave/vortex-vortex interactions (Hall 2018) and vortex stretching (Kline et al. 1967) occurring frequently. A comparison between figures 8 and 9 shows that LEA concentrations are much stronger for the APG case, which indicates a presence of stronger eruption activity. This will inevitably precipitate a more active inner-outer (or viscous-inviscid) interaction within the boundary layer, in a process similar to the autonomous cycle (Jiménez \& Pinelli 1999) or self-sustaining process (Waleffe 1997) in turbulent boundary layers. Compared with a global adverse pressure gradient, transient local APGs are more common in transitional and turbulent boundary layers. In the presence of an HSL or hairpin vortex, a local pressure gradient is induced, which promotes a bursting process and regeneration of hairpin vortices. Although turbulent spots were not observed in our turbulent study, figure 19 reveals that enstrophy concentrations in proximity to the wall are closely related to streamwise vortex tubes in the turbulent boundary layer. However, the local concentrations of high levels of enstrophy are coincident with turbulent-turbulent spots in Wu et al. (2017). This suggests that there is connection between turbulent spots and streamwise vortices in a turbulent boundary layer, and that flow structures primarily originate from the wall.

One commonality between transitional and turbulent boundary layers is the appearance of LSS, as shown in figures 5, 11 and 16. The evolution of timelines for a turbulent spot indicated the development of $\Lambda$-vortices and LSS structures during the propagation of the wave packet. In figure 11(a), a 'bubble-free heart-shaped' region occurs within a HSL between two waves, which was also shown by Acarlar \& Smith (1987a) to appear between a sequence of hairpin heads. In the present study, the HSL is caused by a sequence of wave folds within the wave packet, which also represents folding behaviours of the wave front similar to K-regime transition shown in figure 5 and those shown in Jiang et al. (2020a). In figure $13(b)$, a $\Lambda$-vortex structure is observed to develop prior to the breakdown of the wave packet, which is similar to the observation of Wu et al. (2017). They pointed out that a transitional turbulent spot originates from a $\Lambda$-vortex (and spanwise vortex filament) rather than a streak, but that streaks contribute to the growth of a spot. But in the present study, both the $\Lambda$-vortex and LSS are found to develop from a 3-D wave packet itself. In figure 11, two kinds of LSS are labelled: an LSS1 that develops from an oblique wave, and an LSS2 that is formed between the rotational legs of a $\Lambda$-vortex. Near the wall surface, the LSS structure consists of several 3-D waves (labelled $C$ in figure 12c) (see also the supplementary movie 3), similar to the hypothesis of a SCS in Lee \& Wu (2008). Based on the visualization results and LAE images (figures 11-13), we observed that an LSS, $\Lambda$-vortex and streamwise vortices originate from the deformation of a wave packet. Thus, the final breakdown of the packet is hypothesized to be caused by the interaction between vortices and LSS, and that turbulent spots form in a way of lateral and streamwise regeneration of vortices similar to Haidari \& Smith (1994).

As a middle course between K-regime transition and turbulence, bypass transition holds similarity for both of the flows. Based on the results of the flow visualization and LAE distributions, the development of a 3-D wave and its underlying vortex dynamics are qualitatively the same for K-regime transition (ZPG and APG cases) and bypass transition. Here, a wave-induced model is proposed to describe the process. As a generated 3-D wave travels downstream at a slower speed than the free stream velocity, it moves upward causing a retardation of the outer fluid. This deflection of the outer fluid results in inflections in streamwise and transverse velocities, leading to the development of a 3-D inflectional profile, as depicted in Smith (1984). Note that a tongue-shaped or triangular 
bulge (corresponding to an initial LSS) appears in the near-wall region at this stage. The ejections and sweeps that are stimulated by the 3-D wave lift-up will create a high vorticity concentration (or HSL), which subsequently breaks down, rolling up into discrete hairpin or $\Lambda$-vortices. This process is similar to the advected instability caused by the $3-\mathrm{D}$ separation from a hemisphere or a transverse fluid injection from the wall (Acarlar \& Smith 1987a, $b$; Sabatino \& Rossmann 2016). At this stage, another kind of LSS that is produced by the induction of hairpin vortices becomes dominant in timeline visualizations.

With the development of hairpin vortices, a stronger viscous-inviscid interaction occurs, leading to the breakdown of the 3-D wave packet as well as the regeneration of hairpin vortices (Zhou et al. 1999). As mentioned above, an interaction between vortices and LSS, in a way similar to the self-sustaining process of Waleffe (1997), leads to either a turbulent spot or quasi-streamwise vortices. The spot inception mechanism is by systematic generation of secondary and subsidiary vortices, initiating lateral spreading of the flow deformations, as shown by extensive hydrogen-bubble visualization in Haidari \& Smith (1994). The quasi-streamwise structures in the near-wall region (such as those shown in figure 19) indicate the presence of legs or streamwise vortices generated during the development of a hairpin vortex, which agrees with the LAE results shown in figures 8, 9 and 13. Although not shown in this paper, the regeneration and interaction of hairpin vortices also produces hairpin packets, superstructures and very large-scale motions within the outer layer (Adrian 2007; Marusic \& Monty 2019). The presence of hairpin packets, which originate from the legs of fragmented $\Lambda$ vortices, is found to be instrumental in the breakdown during bypass transition (Wu \& Moin 2009). The wave-induced model provides a logical bridge between the transitional and turbulent boundary layers, employing the viscous-inviscid mechanism within the framework of 3-D wave packet behaviour to explain the observed visualized flow patterns during the flow structure development.

It should be noted that the method of LAE is helpful in revealing the flow physics behind observed visualization patterns. This is different from the method of LAVD, in which a spatial average of vorticity is subtracted at each time step. In the case of wall-bounded shear flows, the vorticity subtraction or deviation may underestimate/overestimate shear strength below/above a certain wall-normal level. The LAE measure may be a superior metric in those cases, since one never observes the mean in an instantaneous snapshot and the LAE field computed within the boundary layer reflects stretching caused by either a background shear field or induced locally by the vortex itself. The isosurface of the LAE may not exactly coincide with vortical structures in the boundary layers. However, Lagrangian-averaged vorticity intensity represented by LAE is able to provide a picture that may illustrate regions where the presence of vortical structures are highly probable. Similar to other vortical identification schemes (Chong et al. 1990; Jeong \& Hussain 1995), the method of LAE is inevitably hampered by an arbitrariness in the selection threshold, thus further effort is required to investigate the enstrophy dynamics revealed using this technique.

\section{Concluding remarks}

In the present study, both transitional and turbulent boundary layers are investigated using Lagrangian tracking methods. The transitional flows investigated includes K-regime transition and bypass transition. Flow structures in K-regime transition with a ZPG and an APG (Hartree parameter $\beta_{H}=-0.062$ ) are first examined using timeline and material surface visualization synthesized from data sets obtained using NPSE. The underlying mechanism that gives rise to the deformation of the material surfaces is also studied 


\section{Three-dimensional wave structure in boundary layers}

using the method of LAE. A comparative study shows that the development process of structures in the APG case is markedly similar to that in a non-gradient (Blasius) boundary layer, i.e. 3 -D wave structures progressively develop into $\Lambda$-vortices, accompanied by multiple folding wave behaviour. However, the structures in the APG flow are more complex and evolve much more rapidly. There are stronger quasi-streamwise vortices that develop beneath the legs of the primary $\Lambda$-vortices in the APG case, which suggests that these vortex legs may precipitate a more active inner-outer (or viscous-inviscid) interaction within the boundary layer. Due to more inflectional boundary layer profiles, a more violent downward sweep behaviour occurs initially for an APG case, indicating a stronger transverse shear layer developing in front of a warped vortex head. Once a $\Lambda$-vortex appears in the APG boundary layer, it is stretched and moves rapidly upward at a steeper inclined angle. It is hypothesized that the amplification and lift-up of a 3-D wave causes the development of high-shear layers at the edge of the WWF, which further evolve into $\Lambda$-vortices. These 3 -D waves, are hypothesized to have their origin in the near-wall flow, creating an interfacial shear layer with the higher-speed outer fluid, similar to SCS (Lee \& Wu 2008; Lee \& Jiang 2019; Jiang et al. 2020a).

For bypass transition, the development of a turbulent spot is calculated on a flat plate at Ma 3 using DNS. Timeline visualization is applied to show both the linear and nonlinear development of wave packets. Within the nonlinear region, both $\Lambda$-vortices and LSS are observed. The breakdown from a wave packet to a turbulent spot is hypothesized to be the result of the growth of a $\Lambda$-vortex due to interaction between the $\Lambda$-vortex and the oblique waves. The LSS near the wall consist of 3-D waves, which are similar to a solition-like coherent structure. The near-wall 3-D wave structure is observed to be the initiator of vortical structures by bypass transition, and plays an important role in the breakdown of wave packets into a turbulent spot.

The 3-D evaluation of the timelines synthesized from a Tomo-PIV data set demonstrates that near-wall fluid moves upward in the centre of an LSS, flanked by the downward movement of outer region fluid at the sides of the streak. The LSS in a turbulent boundary layer appear markedly similar to the results of the transition studies, especially the bypass transition. The present results suggest that an LSS in a turbulent boundary layer may be the result of the development of 3-D wave behaviour. The streamwise vortices observed in our studies are hypothesized to be the result of the amplification and breakdown of the 3-D wave behaviour of the LSS. The similarity of the structures and development in the present transitional and turbulent boundary layer studies supports the concept that the amplification of 3-D waves, developing into vortical flow structures, is a promising scenario for the origin of turbulence production.

Supplementary movies. Supplementary movies are available at https://doi.org/10.1017/jfm.2020.1023.

Acknowledgements. The authors are grateful to anonymous referees for their comments and suggestions which have led to substantial improvement of the paper. We appreciate Dr X. Chen of China Aerodynamics Research and Development Center for providing NPSE data sets for this work. X.Y.J. appreciates discussions with Dr A.K. Gao of Imperial College.

Funding. P.F.L. acknowledges support from the European Research Council (ERC) under the European Union's Horizon 2020 research and innovation grant no. 742480 'Stratified Turbulence And Mixing Processes' (STAMP). This work was supported by the National Natural Science Foundation of China (10921202, 11221061, 11632002, 11521091, 91752202 and 11602005) and National Key Project GJXM92579.

Declaration of interests. The authors report no conflict of interest. 


\section{Author ORCIDs.}

(1) X.Y. Jiang https://orcid.org/0000-0002-3565-6208;

(ㄷ) C.B. Lee https://orcid.org/0000-0003-1552-1346.

\section{Appendix A. Governing equations of DNS}

$$
\begin{aligned}
& \frac{\partial}{\partial t} U+\frac{\partial}{\partial x} f_{1}+\frac{\partial}{\partial y} f_{2}+\frac{\partial}{\partial z} f_{3}=\frac{\partial}{\partial x} V_{1}+\frac{\partial}{\partial y} V_{2}+\frac{\partial}{\partial z} V_{3} \\
& \boldsymbol{U}=[\rho, \rho u, \rho v, \rho w, E]^{\mathrm{T}} . \\
& f_{1}=\left[\rho u, \rho u^{2}+p, \rho u v, \rho u w, u(E+p)\right]^{\mathrm{T}} \text {. } \\
& f_{2}=\left[\rho v, \rho v u, \rho v^{2}+p, \rho v w, v(E+p)\right]^{\mathrm{T}} . \\
& f_{3}=\left[\rho w, \rho w u, \rho w v, \rho w^{2}+p, w(E+p)\right]^{\mathrm{T}} . \\
& V_{1}=\left[0, \sigma_{11}, \sigma_{21}, \sigma_{31}, u \sigma_{11}+v \sigma_{21}+w \sigma_{31}+k \frac{\partial T}{\partial x}\right]^{\mathrm{T}} \text {. } \\
& V_{2}=\left[0, \sigma_{12}, \sigma_{22}, \sigma_{32}, u \sigma_{12}+v \sigma_{22}+w \sigma_{32}+k \frac{\partial T}{\partial y}\right]^{\mathrm{T}} \text {. } \\
& V_{3}=\left[0, \sigma_{13}, \sigma_{23}, \sigma_{33}, u \sigma_{13}+v \sigma_{23}+w \sigma_{33}+k \frac{\partial T}{\partial z}\right]^{\mathrm{T}} \text {. } \\
& p=\frac{1}{\gamma M a^{2}} \rho T, \quad E=\rho\left(C_{V} T+V^{2} / 2\right), \quad k=\frac{C_{p} \mu}{P r} . \\
& C_{p}=\gamma C_{V}, \quad C_{V}=\frac{1}{\gamma(\gamma-1) M a^{2}} . \\
& \sigma_{11}=2 \mu \frac{\partial u}{\partial x}-\frac{2}{3} \mu \operatorname{div}(V), \quad \sigma_{22}=2 \mu \frac{\partial v}{\partial y}-\frac{2}{3} \mu \operatorname{div}(V) . \\
& \sigma_{33}=2 \mu \frac{\partial w}{\partial z}-\frac{2}{3} \mu \operatorname{div}(\boldsymbol{V}), \quad \sigma_{12}=\sigma_{21}=\mu\left\{\frac{\partial u}{\partial y}+\frac{\partial v}{\partial x}\right\} . \\
& \sigma_{13}=\sigma_{21}=\mu\left\{\frac{\partial u}{\partial z}+\frac{\partial w}{\partial x}\right\}, \quad \sigma_{23}=\sigma_{32}=\mu\left\{\frac{\partial v}{\partial z}+\frac{\partial w}{\partial y}\right\} .
\end{aligned}
$$

\section{REFERENCES}

ACARLAR, M.S. \& SMith, C.R. $1987 a$ A study of hairpin vortices in a laminar boundary layer. Part 1. Hairpin vortices generated by a hemisphere protuberance. J. Fluid Mech. 175, 1-41.

ACARLAR, M.S. \& SMITH, C.R. 1987b A study of hairpin vortices in a laminar boundary layer. Part 2. Hairpin vortices generated by fluid injection. J. Fluid Mech. 175, 43-83.

AdRIAN, R.J. 2007 Hairpin vortex organization in wall turbulence. Phys. Fluids 19 (4), 041301.

Asai, M., Konishi, Y., OizUmi, Y. \& Nishioka, M. 2007 Growth and breakdown of low-speed streaks leading to wall turbulence. J. Fluid Mech. 586, 371-396.

Asai, M., Minagawa, M. \& NishiokA, M. 2002 The instability and breakdown of a near-wall low-speed streak. J. Fluid Mech. 455, 289-314. 


\section{Three-dimensional wave structure in boundary layers}

Bertolotti, F.P., Herbert, T. \& Spalart, P.R. 1992 Linear and nonlinear stability of the Blasius boundary-layer. J. Fluid Mech. 242, 441-474.

Borodulin, V.I., Kachanov, Y.S. \& Roschektayev, A.P. 2006 Turbulence production in an APG-boundary-layer transition induced by randomized perturbations. J. Turbul. 7 (8), 1-30.

ChAnG, C.-L. \& MALiK, M.R. 1994 Oblique-mode breakdown and secondary instability in supersonic boundary layers. J. Fluid Mech. 273, 323-360.

CHEN, W. 2013 Numerical simulation of boundary layer transition by combined compact difference method. $\mathrm{PhD}$ thesis, Nanyang Technological University.

Chen, X., ZHU, Y.D. \& LEE, C.B. 2017 Interactions between second mode and low-frequency waves in a hypersonic boundary layer. J. Fluid Mech. 820, 693-735.

Chong, M.S., Perry, A.E. \& CAntwell, B.J. 1990 A general classification of three-dimensional flow fields. Phys. Fluids 2 (5), 765-777.

Deng, S., PAn, C., WANG, J.J. \& He, G. 2018 On the spatial organization of hairpin packets in a turbulent boundary layer at low-to-moderate Reynolds number. J. Fluid Mech. 844, 635-668.

Green, M.A., Rowley, C.W. \& Haller, G. 2007 Detection of Lagrangian coherent structures in three-dimensional turbulence. J. Fluid Mech. 572, 111.

HAIDARI, A.H. \& SMITH, C.R. 1994 The generation and regeneration of single hairpin vortices. J. Fluid Mech. 277, 135-162.

Hall, P. 2018 Vortex-wave interaction arrays: a sustaining mechanism for the log layer? J. Fluid Mech. 850, 46-82.

Haller, G. 2005 An objective definition of a vortex. J. Fluid Mech. 525, 1-26.

Haller, G. 2015 Lagrangian coherent structures. Annu. Rev. Fluid Mech. 47 (1), 137-162.

Haller, G., Hadjighasem, A., Farazmand, M. \& Huhn, F. 2016 Defining coherent vortices objectively from the vorticity. J. Fluid Mech. 795, 136-173.

HALler, G. \& YuAN, G. 2000 Lagrangian coherent structures and mixing in two-dimensional turbulence. Physica D 147, 352-370.

Hama, F.R., Long, J.D. \& Hegarty, J.C. 1957 On transition from laminar to turbulent flow. J. Appl. Phys. 28 (4), 388-394.

HAMA, F.R. \& NutAnT, J. 1963 Detailed flow-field observations in the transition process in a thick boundary layer. In Proceedings of the Heat Transfer and Fluid Mechanics Institute, pp. 77-93. Stanford University Press.

JEONG, J. \& HuSSAIN, F. 1995 On the identification of a vortex. J. Fluid Mech. 285, 69-94.

Jiang, X.Y., Lee, C.B., Chen, X., Smith, C.R. \& Linden, P.F. 2020a Structure evolution at early stage of boundary-layer transition: simulation and experiment. J. Fluid Mech. 890, A11.

JiAng, X.Y., LeE, C.B., Smith, C.R., Chen, J.W. \& Linden, P.F. $2020 b$ Experimental study on low-speed streaks in a turbulent boundary layer at low Reynolds number. J. Fluid Mech. 903, A6.

JiMÉnEZ, J. 2018 Coherent structures in wall-bounded turbulence. J. Fluid Mech. 842, P1.

JimÉnEZ, J. \& PinElli, A. 1999 The autonomous cycle of near-wall turbulence. J. Fluid Mech. 389, 335-359.

KaChanOV, Y.S. 1994 Physical mechanisms of laminar-boundary-layer transition. Annu. Rev. Fluid Mech. $26(1), 411-482$.

Kim, H., Kline, S.J. \& Reynolds, W.C. 1971 The production of turbulence near a smooth wall in a turbulent boundary layer. J. Fluid Mech. 50, 133-60.

Kline, S.J., Reynolds, W.C., Schraub, F.A. \& Runstadler, P.W. 1967 The structure of turbulent boundary layers. J. Fluid Mech. 30 (4), 741-773.

KLOKER, M. \& FASEL, H. 1995 Direct numerical simulation of boundary-layer transition with strong adverse pressure gradient. In Laminar-Turbulent Transition (ed. R. Kobayashi), pp. 481-488. Springer.

KRISHNAN, L. \& SANDHAM, N.D. 2006 Effect of mach number on the structure of turbulent spots. J. Fluid Mech. 566, 225-234.

LAURIEN, E. \& KLEISER, L. 1989 Numerical simulation of boundary-layer transition and transition control. J. Fluid Mech. 199, 403-440.

LEE, C.B. 1998 New features of CS solitons and the formation of vortices. Phys. Lett. A 247 (6), $397-402$.

LEE, C.B. 2000 Possible universal transitional scenario in a flat plate boundary layer: measurement and visualization. Phys. Rev. E 62 (3), 3659-3670.

LeE, C.B. \& JiAng, X. 2019 Flow structures in transitional and turbulent boundary layers. Phys. Fluids 31 (11), 111301.

LEE, C.B. \& LI, R.Q. 2007 Dominant structure for turbulent production in a transitional boundary layer. J. Turbul. 8 (55), 1-34.

LEE, C.B. \& Wu, J.Z. 2008 Transition in wall-bounded flows. Appl. Mech. Rev. 61, 030802.

LI, X.L., FU, D.X. \& MA, Y.W. 2008 Direct numerical simulation of hypersonic boundary-layer transition over a blunt cone. AIAA J. 46 (11), 2899-2913. 


\section{X.Y. Jiang, D.W. Gu, C.B. Lee, C.R. Smith and P.F. Linden}

LYNCH, K.P. \& SCARANO, F. 2015 An efficient and accurate approach to MTE-MART for time-resolved tomographic PIV. Exp. Fluids 56 (3), 66.

MARUSIC, I. 2009 Unravelling turbulence near walls. J. Fluid Mech. 630, 1-4.

Marusic, I. \& Monty, J.P. 2019 Attached eddy model of wall turbulence. Annu. Rev. Fluid Mech. 51 (1), 49-74.

MusKer, A.J. 1979 Explicit expression for the smooth wall velocity distribution in a turbulent boundary layer. AIAA J. 17 (6), 655-657.

Neamtu-Halic, M.M., Krug, D., Mollicone, J.-P., Van Reeuwijk, M., Haller, G. \& HolzNER, M. 2020 Connecting the time evolution of the turbulence interface to coherent structures. J. Fluid Mech. 898, A3.

Park, G.I., Wallace, J.M., Wu, X.H. \& Moin, P. 2012 Boundary layer turbulence in transitional and developed states. Phys. Fluids 24 (3), 035105.

REMPFER, D. 2003 Low-dimensional modeling and numerical simulation of transition in simple shear flows. Annu. Rev. Fluid Mech. 35 (1), 229-265.

RisT, U. \& FASEL, H. 1995 Direct numerical simulation of controlled transition in a flat-plate boundary layer. J. Fluid Mech. 298, 211-248.

Robinson, S.K. 1991 Coherent motions in the turbulent boundary layer. Annu. Rev. Fluid Mech. 23 (1), 601-639.

Sabatino, D.R. \& Rossmann, T. 2016 Tomographic PIV measurements of a regenerating hairpin vortex. Exp. Fluids 57 (1), 1-13.

Sayadi, T., Hamman, C.W. \& Moin, P. 2013 Direct numerical simulation of complete h-type and k-type transitions with implications for the dynamics of turbulent boundary layers. J. Fluid Mech. 724, 480-509.

SAyAdi, T., Schmid, P.J., Nichols, J.W. \& Moin, P. 2014 Reduced-order representation of near-wall structures in the late transitional boundary layer. J. Fluid Mech. 748, 278-301.

Schoppa, W. \& Hussain, F. 2002 Coherent structure generation in near-wall turbulence. J. Fluid Mech. 453, 57-108.

SMith, C.R. 1984 A synthesized model of the near-wall behavior in turbulent boundary layers. In Proceedings of the 8th Symposium of Turbulence (ed. G.K. Pattersonand \& J.L. Zakin), pp. 1-27. University of Missouri-Rolla.

Smith, C.R. \& MetzleR, S.P. 1983 The characteristics of low-speed streaks in the near-wall region of a turbulent boundary layer. J. Fluid Mech. 129, 27-54.

Smith, C.R., Walker, J.D.A., Haidari, A.H. \& Sobrun, U. 1991 On the dynamics of near-wall turbulence. Phil. Trans. R. Soc. Lond. A 336 (1641), 131-175.

Tian, S.L., GaO, Y.S., Dong, X.R. \& LiU, C.Q. 2018 Definitions of vortex vector and vortex. J. Fluid Mech. 849, 312-339.

WALEFFE, F. 1997 On a self-sustaining process in shear flows. Phys. Fluids 9 (4), 883.

WIENEKE, B. 2008 Volume self-calibration for 3D particle image velocimetry. Exp. Fluids 45 (4), $549-556$.

WortmAnN, F.X. 1981 Boundary-layer waves and transition. In Advances in Fluid Mechanics (ed. E. Krause), pp. 268-279. Springer.

WU, Y. 2014 A study of energetic large-scale structures in turbulent boundary layer. Phys. Fluids 26 (4), 045113.

Wu, J.-Z., MA, H.-Y. \& ZHou, M.-D. 2006 Vorticity and Vortex Dynamics. Springer.

WU, X.H. \& MoIN, P. 2009 Direct numerical simulation of turbulence in a nominally zero-pressure-gradient flat-plate boundary layer. J. Fluid Mech. 630, 5-41.

Wu, X.H., MOIN, P. \& ADRIAN, R.J. 2020 Laminar to fully turbulent flow in a pipe: scalar patches, structural duality of turbulent spots and transitional overshoot. J. Fluid Mech. 896, A9.

Wu, X., Moin, P., Wallace, J.M., Skarda, J., Lozano-Duran, A. \& Hickey, J.-P. 2017 Transitional-turbulent spots and turbulent-turbulent spots in boundary layers. Proc. Natl Acad. Sci. USA 114 (27), E5292-E5299.

ZHAO, Y., YANG, Y. \& CHEN, S. 2016 Evolution of material surfaces in the temporal transition in channel flow. J. Fluid Mech. 793, 840-876.

Zhou, J., Adrian, R.J., Balachandar, S. \& Kendall, T.M. 1999 Mechanisms for generating coherent packets of hairpin vortices in channel flow. J. Fluid Mech. 387, 353-396.

Zhu, Y., Lee, C., Chen, X., Wu, J., Chen, S. \& Gad-el HaK, M. 2018 Newly identified principle for aerodynamic heating in hypersonic flows. J. Fluid Mech. 855, 152-180. 